\title{
OPEN Microbiome and metabolome profiles of high screen time in a cohort of healthy college students
}

\author{
Paniz Jasbi ${ }^{1,8}$, Alex E. Mohr ${ }^{1,8}$, Xiaojian Shi ${ }^{1,2}$, Tara Mahmood ${ }^{1,3}$, Qiyun Zhu ${ }^{4,5}$, \\ Meg Bruening ${ }^{1}$, Haiwei $\mathrm{Gu}^{1,6 \bowtie}$ \& Corrie Whisner ${ }^{1,7 凶}$
}

As screens are increasingly integrated into every facet of modern life, there is growing concern over the potential effects of high screen time. Previous studies have largely utilized self-report data on mood and behavioral aspects of screen time, and no molecular theory has yet been developed. In this study, we explored the fecal microbiome and metabolome of a diverse group of 60 college students, classified by high ( $\geq 75 \mathrm{~min} /$ day) or low (0-75 min/day) self-reported screen time using 16S rRNA amplicon sequencing, targeted liquid chromatography-tandem mass spectrometry, and targeted detection of short-chain fatty acids using gas chromatography-mass spectrometry. Several key taxa and metabolites were significantly altered between groups and found to be highly co-occurrent. Results of pathway and enzyme enrichment analyses were synthesized to articulate an integrated hypothesis indicating widespread mitochondrial dysfunction and aberrant amino acid metabolism. High screen time was also predicted to be significantly associated with type I diabetes, obesity, chronic fatigue syndrome, and various manifestations of inflammatory bowel. This is the first-ever study to report the effects of high screen time at the molecular level, and these results provide a datadriven hypothesis for future experimental research.

Screen time, or the time spent using a phone or tablet device, computer, television, or game console, is a matter of increasing public and scientific concern ${ }^{1}$. Electronic screen use has expanded as new technologies are increasingly integrated across all domains of life, including work, entertainment, physical activity, education, travel, finance, and even romance ${ }^{2}$. Screen-based activities are particularly prevalent in younger demographics ${ }^{3}$, with data suggesting significant increases in daily average screen time from infancy to adolescence. In 2013, daily average screen time for children under three in the US was estimated to be 42 minutes ${ }^{4}$, while a 2014 survey of the same measure for Australian children and adolescents (8-18 years old) was estimated to be $7 \mathrm{~h}$ and 38 minutes ${ }^{5}$. Estimates of screen time for college students are limited and range widely from 2.8 to $11.6 \mathrm{~h} \mathrm{per} \mathrm{day}^{6}$, and show that female students and physically active students report significantly fewer minutes of total screen time than their male and physically inactive counterparts, respectively ${ }^{7}$.

As widespread use of screen-based devices has grown, so have concerns over their possible impact on physical and mental health. However, current research is largely focused on the relationship between screen-based activity and mental/behavioral health issues. For instance, results from longitudinal cohort studies have provided evidence that higher levels of daily screen time may impede childhood development ${ }^{8}$ and have been positively associated with depression during adolescence ${ }^{9}$. Other problematic areas include reductions in sleep quality and physical activity ${ }^{10}$, as well as increased consumption and desire for alcohol and sweets ${ }^{11}$. These findings have spurred recent guidelines on limiting screen time. Stringent limits on screen use for infants and children under five have been expounded by both the World Health Organization (WHO) ${ }^{12}$ and the American Academy of Pediatrics (AAP) $)^{13}$ : for all children under 5, both the WHO and AAP recommend no more than 60 min of screen

${ }^{1}$ College of Health Solutions, Arizona State University, Phoenix, AZ, USA. ${ }^{2}$ Systems Biology Institute, Yale University, West Haven, CT, USA. ${ }^{3}$ Department of Nutrition, Texas A\&M University, College Station, TX, USA. ${ }^{4}$ School of Life Sciences, Arizona State University, Tempe, AZ, USA. ${ }^{5}$ Biodesign Center for Fundamental and Applied Microbiomics, Arizona State University, Tempe, AZ, USA. ${ }^{6}$ Center for Translational Science, Florida International University, Port St. Lucie, FL, USA. ${ }^{7}$ Biodesign Institute Center for Health Through Microbiomes, Arizona State University, Tempe, AZ, USA. ${ }^{8}$ These authors contributed equally: Paniz Jasbi and Alex E. Mohr. ${ }^{\varpi}$ email: hgu@fiu.edu; cwhisner@asu.edu 
time per day. For children and adolescents ages 5 to 18, the AAP recommends regulating the quality of screen content rather than quantity, with specific recommendations related to screen use before bed and limiting the number of screens in the living environment ${ }^{13}$. Although there is no consensus on a safe amount of screen time for adults, associations between well-being and digital technology use show deleterious effects on individuals engaging in more than 120 min of daily screen time ${ }^{2}$.

Far fewer studies have explored risk factors and consequences of screen time on more complex biological data. One study examining consumption of screen-based media in preschool-age children demonstrated significant correlations between higher screen use and measures of white matter tract demyelination ${ }^{14}$. Another study involving magnetic resonance imaging demonstrated a strong, positive association between brain connectivity and time spent reading books whereas a strong, negative association was observed between length of exposure to screen-based media and degree of neuronal arborization ${ }^{15}$. Furthermore, it appears as though physical activity does not compensate for the adverse effects of screen time on the microstructure of the central nervous system $^{16,17}$. No study to date has investigated the potential effects of screen time using a systems biology approach.

Comprised of thousands of different bacterial taxa as well as various archaea, eukaryotic microbes and viruses, the gut microbiota (GM) is now understood to play a significant role in human health ${ }^{18}$. Correspondingly, advances in next-generation sequencing technology have rendered 16S rRNA amplicon sequencing an attractive platform for monitoring changes in the $\mathrm{GM}^{19}$ given its high-throughput parallel sequencing, rapid time-to-analysis, and feasibility ${ }^{20}$. As an internal bioreactor, the GM has tremendous functional capacity ${ }^{21}$, producing a broad suite of metabolites that have varying effects on host health ${ }^{22}$. Like the GM, metabolomic profiles also present a unique, person-specific signature and are heavily predicted by host-associated characteristics and environmental factors ${ }^{23}$. Defined as the complete suite of metabolites (small molecules $<2000 \mathrm{Da}$ ) present in a biological system, the metabolome can complement microbiomic readouts and is impacted by similar host-associated characteristics ${ }^{24}$. In particular, aqueous metabolites and short-chain fatty acids (SCFAs) play key roles in host-microbe interaction ${ }^{24}$ and bacterial signaling ${ }^{25}$, respectively. For instance, aqueous fecal and plasma metabolites show strong, significant associations to gut microbial diversity ${ }^{24}$ and SCFAs produced by bacterial fermentation of dietary fibers and resistant starch in the colon may enable cell signaling through surface G-protein coupled receptors ${ }^{25}$. Integrating omics-based data is becoming increasingly feasible and offers important biological insight ${ }^{26}$. The GM and associated metabolic output can also be influenced downstream from host behavior including diet, physical activity, and sedentary time ${ }^{27}$.

Previously, Whisner et al. (2018) reported that racially/ethnically diverse first year college students displayed associations of physical activity and screen time with the $\mathrm{GM}^{27}$. This population transitions from family-living to independent living conditions where many new behaviors are instilled, while others are eliminated ${ }^{28}$. The GM in adolescence exhibits greater interpersonal variation and lower bacterial diversity compared to adults, which may promote a more malleable biota ${ }^{29}$. During such a formative period, dietary intake, and health behaviors, including physical activity and sedentarism (e.g., screen time), may allow for greater and lasting microbial shifts. Given the increased prevalence of screen time and the critical formalization of the GM in this age group, we aimed to explore potential differences in the fecal microbiome and metabolome by screen time in a subset of the DevilWaste project cohort of college students $(n=60)^{27}$. Specifically, we performed a cut on self-reported screen time, to compare participants by low (0-75 min/day) vs. high ( $\geq 75 \mathrm{~min} /$ day) screen time using $16 \mathrm{~S}$ rRNA amplicon sequencing in addition to targeted liquid chromatography-tandem mass spectrometry (LC-MS/MS) profiling of aqueous metabolites and targeted gas chromatography (GC)-MS profiling of short-chain fatty acids (SCFAs).

\section{Results}

Study design and participant characteristics. The current study is a cross-sectional investigation of metabolomic and microbiomic differences in a cohort of diverse college students; an overview of the analytical workflow is provided in Fig. 1. A total of 60 participants provided a fecal sample and dietary recall, moderate and vigorous physical activity (MVPA), and screen time data (Table 1). The overall mean percentage of kilocalories from carbohydrate, protein, and fat were $46.0 \pm 17.9 \%, 16.9 \pm 9.3 \%$, and $37.1 \pm 14.7 \%$, respectively (Table 1 ). Both protein and carbohydrate consumption were within the acceptable macronutrient distribution range (AMDR) of $10-35 \%$ and $45-65 \%$, respectively, while the mean fat consumption fell slightly outside the AMDR range of $20-35 \%$. The mean self-reported daily intake of sugar consumed was $75.12 \pm 46.13 \mathrm{~g} /$ day. Mean daily consumption of dietary fiber for males $(n=20)$ and females $(n=40)$ was $12.21 \pm 5.36 \mathrm{~g} /$ day and $12.70 \pm 5.64 \mathrm{~g} / \mathrm{day}$, respectively, which fell below the recommended Adequate Intake for both males ( $38 \mathrm{~g} /$ day) and females (25-26 g/day). No significant difference in self-reported intake (g) of carbohydrates, sugar, fiber, protein, or fat was overserved between high and low screen time groups. Age, sex, body mass index (BMI), and self-reported MVPA did not differ significantly by screen-time classification. Self-reported screen time and MVPA were not significantly correlated (Spearman's $\rho=-0.083, p=0.530$ ). An initial principal component analysis (PCA) was performed between high and low screen time groups using the entire set of captured metabolites and GM features (Supplementary Fig. S1). The first two components accounted for approximately $75 \%$ of all variance, and analysis of 95\% confidence intervals (CIs) showed two potential outliers (DW40 and DW100, one from each study group) which were removed from further analyses upon confirming non-ignorable missingness of metabolomic data.

Diversity metrics did not differ by screen time classification. Alpha diversity is an important metric commonly reported in the microbiome literature, and different indices are needed to assess various aspects of within-sample diversity such as richness, evenness, and dominance. Therefore, we have comprehensively reported the most relevant alpha diversity indices in Fig. 2 and Supplementary Table S1 to ensure maximum transparency. When controlling for BMI, age, MVPA, and sex as covariates, no significant differences in alpha diversity measures were observed between high and low screen time groups (observed features, Faith's PD, Pie- 

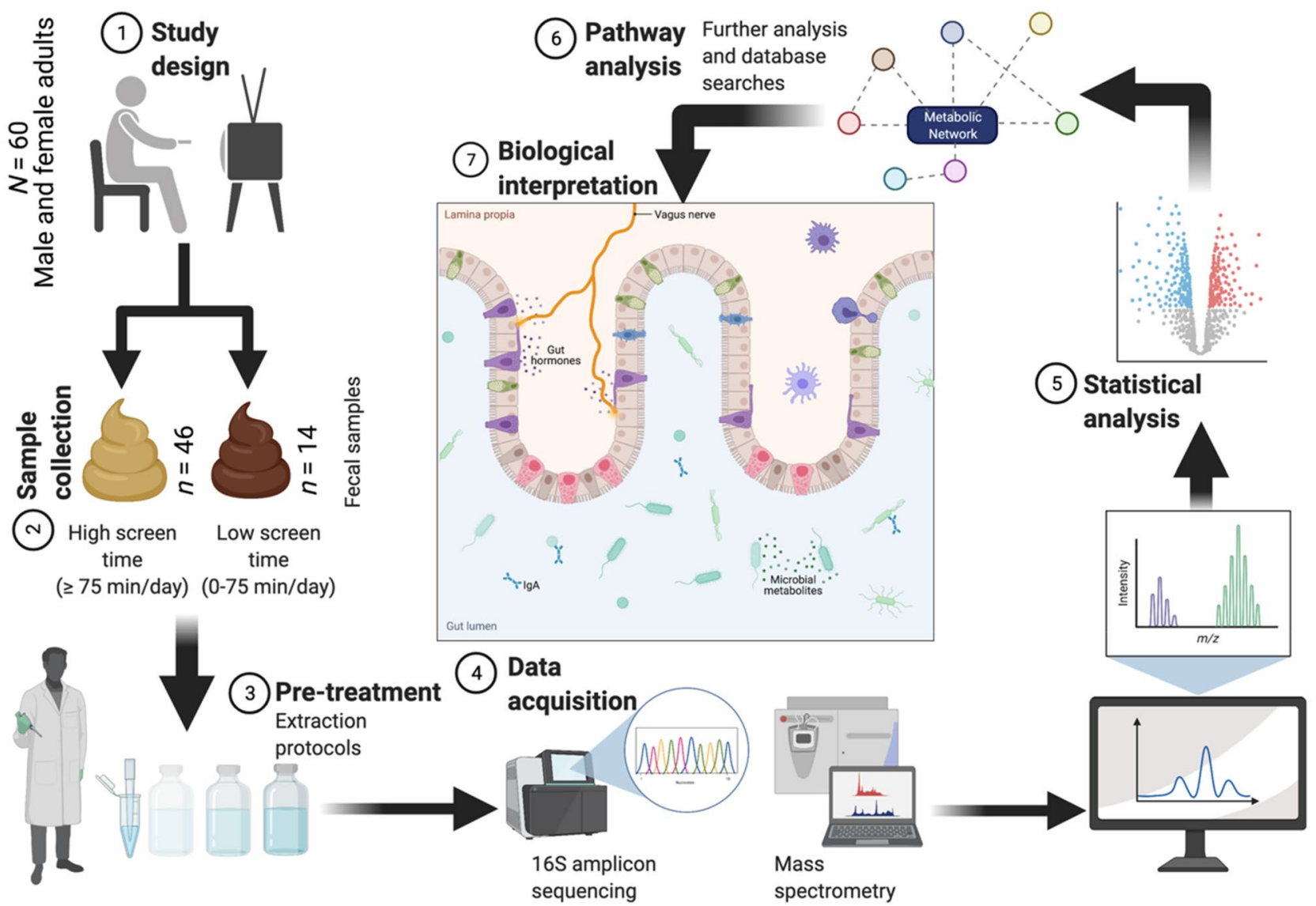

Figure 1. Overview of analytical workflow from study design to biological interpretation. Created with BioRender.com.

\begin{tabular}{|c|c|c|c|}
\hline & Total $(n=60)$ & Low $(n=14)$ & High $(n=46)$ \\
\hline Age, mean $\pm S D$ & $18.5 \pm 0.7$ & $18.6 \pm 0.9$ & $18.4 \pm 0.6$ \\
\hline \multicolumn{4}{|l|}{ Sex, \% (n) } \\
\hline Male & $33.3(20)$ & $42.9(6)$ & $30.4(14)$ \\
\hline Female & $66.7(40)$ & $57.2(8)$ & $69.6(32)$ \\
\hline \multicolumn{4}{|l|}{ Race/ethnicity, \% ( $n$ ) } \\
\hline Hispanic & $23.3(14)$ & $21.4(3)$ & $23.9(11)$ \\
\hline White & $43.3(26)$ & $57.1(8)$ & $39.1(18)$ \\
\hline Other & $33.3(20)$ & $21.4(3)$ & $37.0(17)$ \\
\hline Body Mass Index $\left(\mathrm{kg} / \mathrm{m}^{2}\right)$, mean $\pm \mathrm{SD}$ & $24.4 \pm 5.9$ & $23.3 \pm 3.4$ & $24.8 \pm 6.5$ \\
\hline$<18.5 \mathrm{~kg} / \mathrm{m}^{2} \%(n)$ & $3.3(2)$ & $0(0)$ & $4.3(2)$ \\
\hline $18.5-24.9 \mathrm{~kg} / \mathrm{m}^{2} \%(n)$ & $63.3(38)$ & $71.4(10)$ & $60.9(28)$ \\
\hline $25.0-29.9 \mathrm{~kg} / \mathrm{m}^{2} \%(n)$ & $18.3(11)$ & $21.4(3)$ & $17.4(8)$ \\
\hline$\geq 30.0 \mathrm{~kg} / \mathrm{m}^{2} \%(n)$ & $15.0(9)$ & $7.2(1)$ & $17.4(8)$ \\
\hline Screen time (min/day), median (IQR) & $195.0(195.0,315.0)$ & $15.0(15.0,56.6)$ & $195.0(195.0,360.0)$ \\
\hline Moderate-to-vigorous physical activity (min/day), median (IQR) & $52.2(25.7,77.1)$ & $58.9(26.2,61.6)$ & $45.0(25.7,77.1)$ \\
\hline Diet, mean \pm SD & $1614.2 \pm 589.3$ & $1546.2 \pm 703.5$ & $1634.6 \pm 559.2$ \\
\hline Carbohydrates (g) & $186.1 \pm 72.5$ & $169.0 \pm 61.1$ & $191.3 \pm 75.5$ \\
\hline Sugar $(g)$ & $75.1 \pm 46.1$ & $61.6 \pm 34.2$ & $79.2 \pm 48.8$ \\
\hline Fiber (g) & $12.6 \pm 5.5$ & $11.2 \pm 5.5$ & $13.0 \pm 5.4$ \\
\hline Protein $(\mathrm{g})$ & $68.3 \pm 37.7$ & $75.2 \pm 64.3$ & $66.2 \pm 26.0$ \\
\hline Fat (g) & $66.5 \pm 26.5$ & $63.9 \pm 33.6$ & $66.4 \pm 24.4$ \\
\hline
\end{tabular}

Table 1. Participant characteristics by screen time classification. IQR interquartile range, $S D$ standard deviation. 

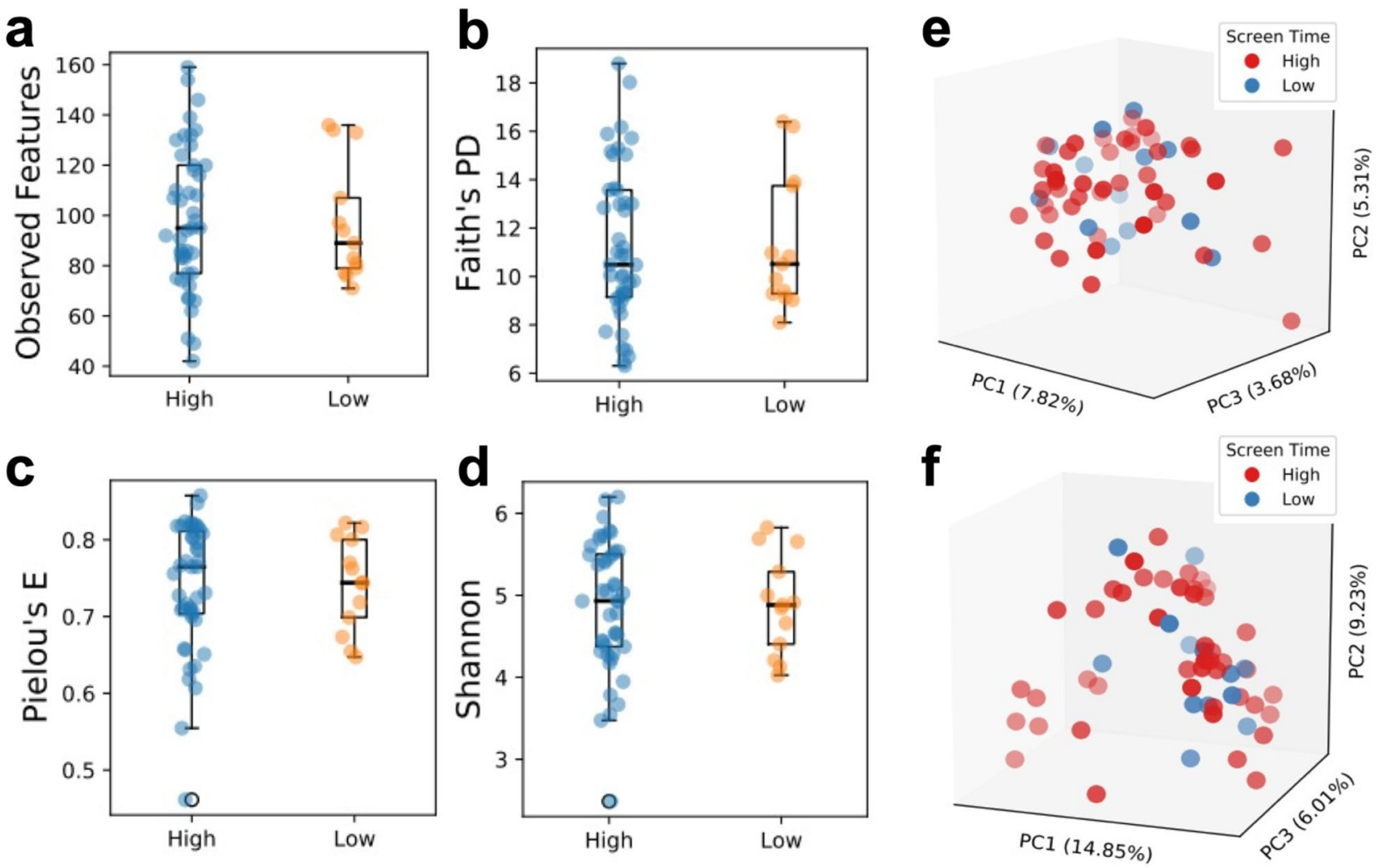

Figure 2. Comparison of alpha and beta diversity metrics between high and low screen time classifications. Boxplots for alpha diversity metrics: (a) observed features (unweighted amplicon sequence variants). (b) Faith's PD (phylogenetic diversity). (c) Pielou's E (species evenness). (d) Shannon index (weighted proportional abundances). PCoA plots for beta diversity metrics: (e) Jaccard. (f) Bray-Curtis. Weighted and unweighted UniFrac distance matrices not displayed.

lou's E, and Shannon index, $p \geq 0.539$ ). These findings suggest that screen time was not associated with GM richness or evenness in this cohort of college students (Supplementary Table S1; Fig. 2a-d). Adonis analysis with the same covariates revealed no significant differences between high and low screen time for beta diversity metrics (Jaccard, Bray Curtis, Unweighted UniFrac, and Weighted UniFrac $p \geq 0.219$; Supplementary Table S1; Fig. 2e-f).

Analysis of gut microbiome composition reveals differential abundances in key microbes between groups. After quality control and filtering of any samples with fewer features than our rarefaction threshold $(10,000)$ and low abundance/low prevalence amplicon sequence variants (ASVs) using the qiime2feature-table plugin with the filter-samples method, we generated and provided taxonomy assignments for 247 features at the genus level (kingdom: 2; phylum: 23; class: 44; order: 69; family: 120; genus: 247). A heatmap of the core microbiome showing prevalence by detection threshold (relative abundance \%) at the genus level is shown in Supplementary Fig. S2. Of the top 15 most prevalent genera, we noted several biologically relevant taxa including, Bacteroides, Prevotella, Faecalibacterium, Roseburia, Alistipes, and Akkermansia (Fig. 3a). To reveal salient inter-community niche feature importance based on composition, we used DEICODE, a form of Aitchison distance that is robust to high levels of sparsity ${ }^{30}$. The output is visualized as a robust Aitchison PCA (Fig. 3b), based on the feature table. Upon performing an Adonis test with BMI, age, MVPA, and sex as covariates, no significance was detected between high and low screen time groups $\left(F=0.391, R^{2}=0.008, p=0.678\right)$, though we did identify several taxa that were correlated with screen time classification including Bacteroides, Prevotella, and Roseburia. A taxonomy bar plot of individual subjects at the genus level by high and low screen time is provided as Fig. 3c.

To better identify specific microbes associated with screen time classification, we used Songbird, a compositionally aware differential abundance method which provides rankings of features based on their log fold change while accounting for relevant covariates ${ }^{31}$. As before, we controlled for BMI, age, MVPA, and sex as covariates and selected the 20 highest ("set 1," Supplementary Table S2)- and 20 lowest ("set 2," Supplementary Table S2)ranked ASV s associated with screen time classification. Next, we used Qurro ${ }^{32}$ to compute the log ratio of these sets of taxa (Fig. 4a). Comparing the ratios of taxa in this way mitigates bias from the unknown total microbial load in each sample and taking the log of this ratio gives equal weight to relative increases and decreases of $\operatorname{taxa}^{31}$. Evaluation of the Songbird model for high/low screen time classification against a baseline model showed exponential decay and a stable plateau, though a $Q^{2}$ value of -0.10 was produced, suggesting a potential for overfitting related to the differences between classifications. Comparing the log ratio of the two sets, we noted the 
a
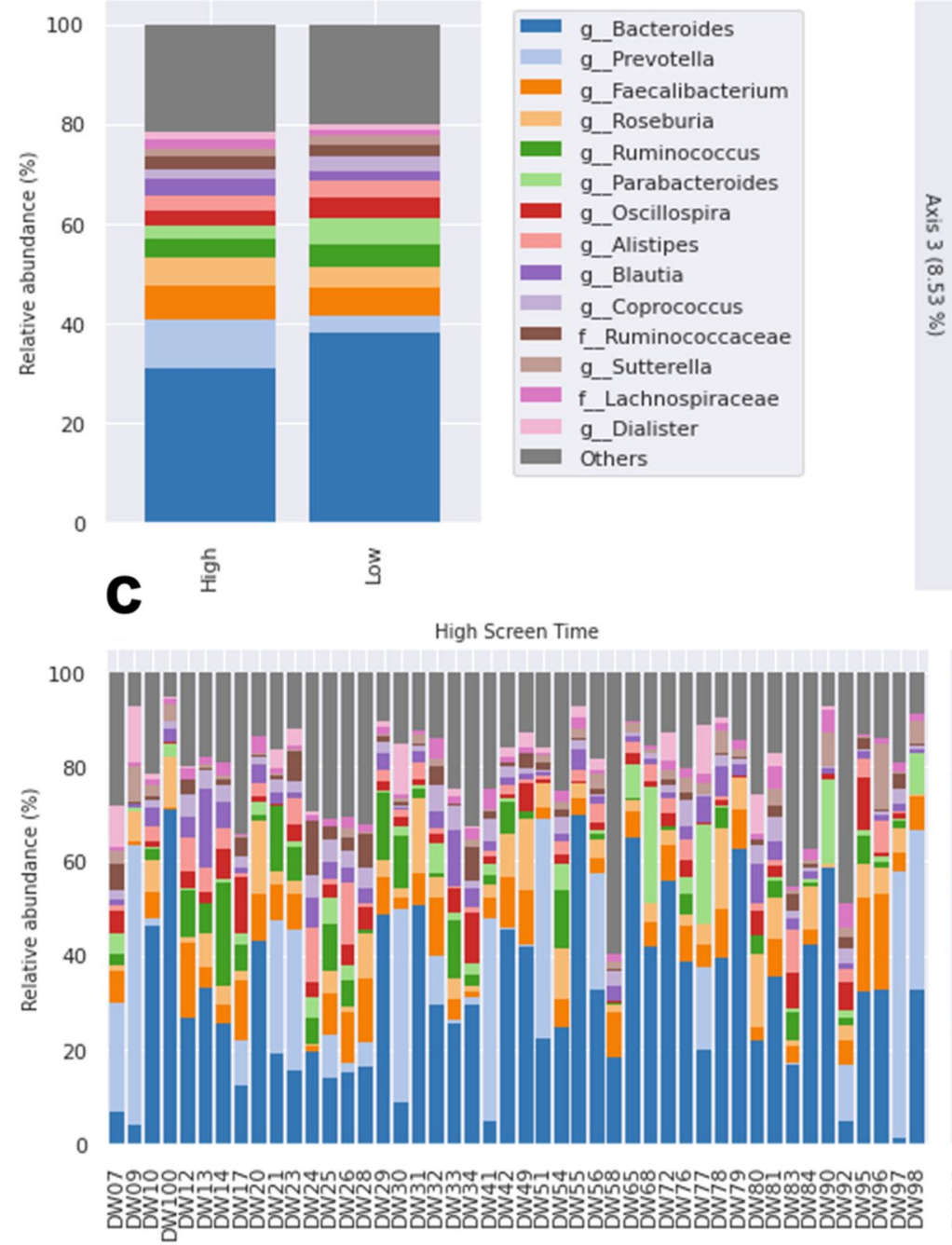

b

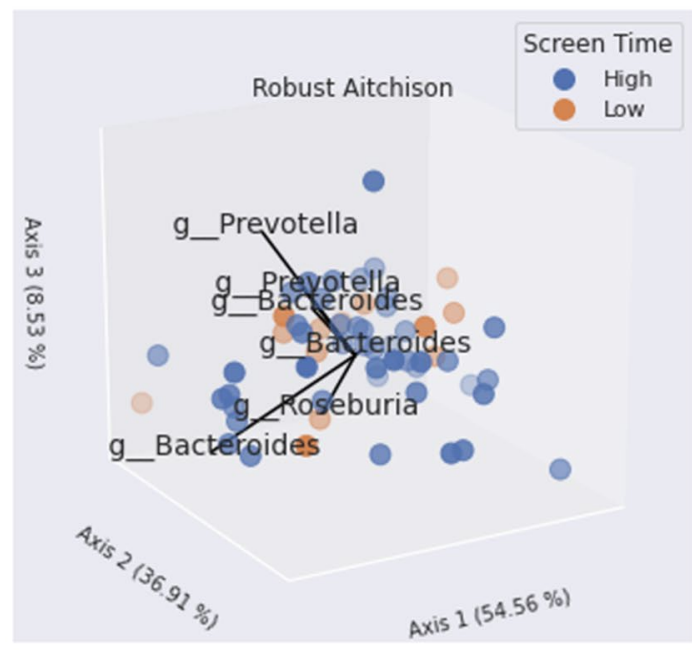

Low Screen Time
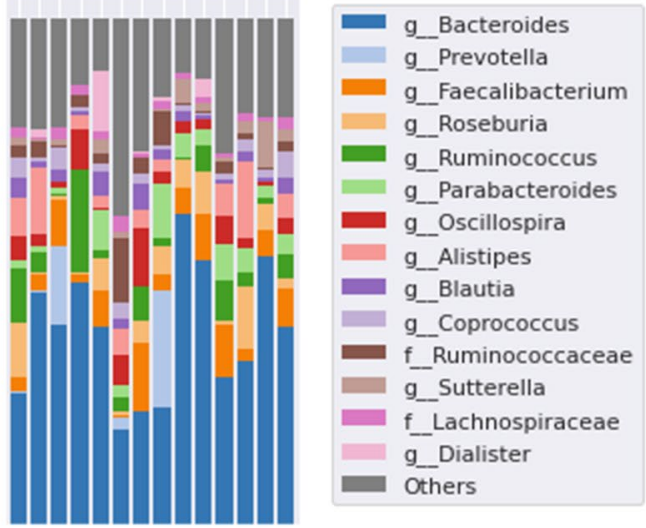

nennenONONMGNA

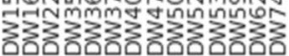

Figure 3. Taxonomic analysis of microbiota between groups. (a) Taxonomy bar plot of the top 15 most abundant taxa at the genus level for both groups $(n=60)$. Less abundant taxa, representing $\sim 19.5 \%$ of relative abundance, are not displayed. (b) Robust Aitchison PCA generated from DEICODE showing taxa (arrows) correlated with screen time classification $(n=58)$. (c) Taxonomy bar plot of individual subjects at the genus level by high and low screen time groups $(n=60)$. Less abundant taxa are not displayed. Note, where resolution at the genus level was not possible taxa are described at the lowest feature level obtained (i.e., $f=$ family).

high screen time group had a significantly greater log ratio of set 2 compared to set 1 (Fig. $4 \mathrm{~b} ; p=0.002$, Cohen's $d=1.89$ ), suggesting that high screen time was more strongly associated with the genera Prevotella, Veillonella, Bacteroides, Lachnospira, Coprococcus, and Ruminococcus. In contrast, low screen time was more associated with the genera Bacteroides, Akkermansia, Alistipes, Ruminococcus, Sutterella, Oscillospira, and Methanobrevibacter. To compare these microbial abundances in a compositionally coherent way, we calculated a log ratio with abundances of several high screen time-specific taxa in the numerator and Ruminococcaceae (genus level not defined) abundance in the denominator, which Songbird multinomial regression identified as the taxa most associated with low screen time (Fig. 4c-f). Most of these comparisons were not significant, although the high screen time classification showed a significantly greater ratio of Peptostreptococcaceae/Runimococcaceae compared to low screen time $(p=0.036)$. High screen time also showed a significantly greater ratio of Bacteroides/Akkermansia $(p=0.044)$, but not Prevotella/Bacteroides $(p=0.518)$, compared to low screen time.

Predicted functional profile of the gut microbiome differs by screen time. The Phylogenetic Investigation of Communities by Reconstruction of Unobserved States 2 (PICRUSt 2) pipeline ${ }^{33}$ was used to infer the functional profile of the GM based on $16 \mathrm{~S}$ amplicon sequencing data. The Kyoto Encyclopedia of Genes and Genomes (KEGG) ${ }^{34}$ outputs were analyzed and illustrated with statistical analysis of the taxonomic and functional profiles. PCA revealed appreciable differences in the predicted functional composition of the GM among the two screen time classifications (e.g., variances accounted by PC 1 and PC 2 were 39.0\% and 


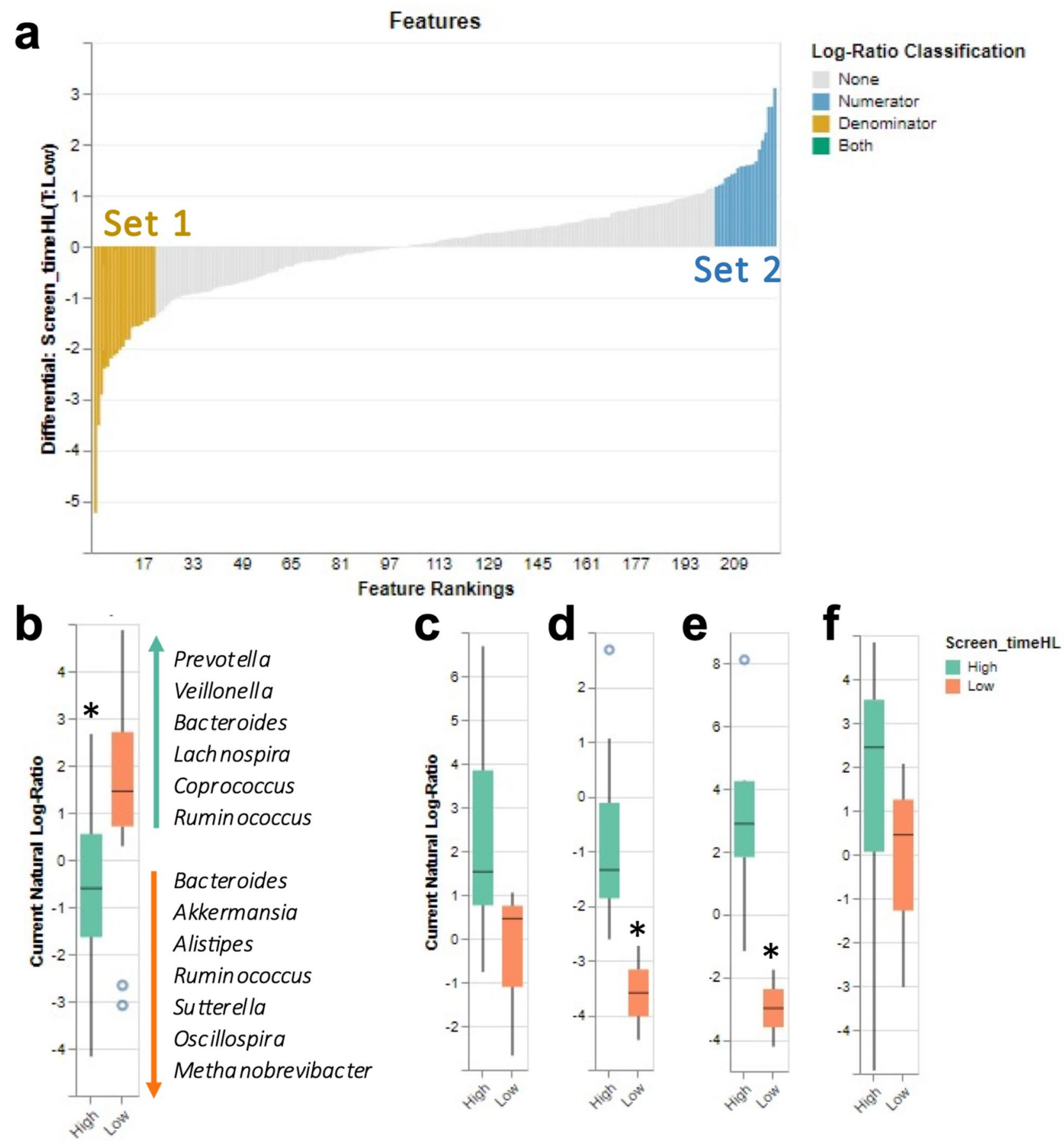

Figure 4. Differential analysis of log ratio classification. (a) Comparison of log ratio of the 20 highest and 20 lowest ranked ASVs associated with screen time classification. (b) High screen time classification had a significantly greater log ratio of set 2 compared to set 1 ( $t$-test); (c) Prevotella//Runimococcaceae (MannWhitney $U$ test); (d) Peptostreptococcaceae/Runimococcaceae (Mann-Whitney $U$ test); (e) Bacteroides/ Akkermansia (Mann-Whitney $U$ test); (f) Prevotella/Bacteroides (Mann-Whitney $U$ test). ${ }^{*} p<0.05$.

$12.5 \%$, respectively) (Supplementary Fig. S3a). A convergence summary of the predicted metabolic pathways also showed some differential enrichment between the two classifications (Supplementary Fig. S3b). Overall, there were 52 enzymes at KEGG level 3 with significant differences in enrichment between low and high screen time classification (Fig. 5). The main differential enzymes between high and low screen time included glutaconate CoA-transferase $(q=0.013)$, protoporphyrinogen oxidase $(q=0.021)$, phosphoenolpyruvate carboxylase $(q=0.022)$, levanase $(q=0.027)$, methylaspartate mutase $(q=0.028)$, NADP + transhydrogenase $(q=0.030)$, glutamate dehydrogenase $(q=0.040)$, and dodecanoyl-ACP hydrolase $(q=0.045)$.

Taxon set enrichment analysis (TSEA) was also performed using MicrobiomeAnalyst ${ }^{35}$. Predicted functional profiles of host-intrinsic factors such as disease were analyzed using 239 taxon sets, whereas host-extrinsic factors 

ino-2-methylpropionate transaminase Phosphogluconate dehydratase $\mathbf{P}$ Gluconate 2-dehydrogenase P Cyanophycinase $P$ Cyanophycin synthase (L-arginine-adding) $P$ Cyanophycin synthase ( $L$-aspartate-adding) $P$ Chorismate lyase $\mathbf{P}$ Methylthioribulose 1-phosphate dehydratase | [Isocitrate dehydrogenase $(\operatorname{NADP}(+))$ ] kinase P 3-phytase I Malate dehydrogenase (quinone) $\mathrm{P}$ Phosphomevalonate kinase $P$ Phosphoribulokinase $\boldsymbol{P}$ Xenobiotic-transporting ATPase P

Glutaconate CoA-transferase $\square$ Serine 3-dehydrogenase $(\operatorname{NADP}(+))$ | Isocitrate lyase $P$

Coenzyme gamma-F420-2:alpha-L-glutamate ligase P

2-methylcitrate dehydratase |

"Quinoprotein glucose dehydrogenase (PQQ, quinone)" P Acireductone synthase I Pyruvate oxidase $\mathbf{p}$ Dihydropyrimidine dehydrogenase $(\operatorname{NADP}(+))$ | Protoporphyrinogen oxidase Phospholipase $\mathrm{CP}$ Nitrite reductase (NADH) Phosphoenolpyruvate carboxylase Acetoacetate decarboxylase $\mathbf{P}$ Methylisocitrate lyase $P$ $\mathrm{N}$-acetylglucosaminyl-diphospho-decaprenol L-rhamno... I 4-alpha- $\mathrm{N}$-acetylgalactosaminyltransferase | Levanase E

3-hydroxybutyryl-CoA epimerase $P$ Fatty-acid peroxygenase ? Methylaspartate mutase

Acireductone dioxygenase ( $\mathrm{Fe}(2+)$-requiring) I Acireductone dioxygenase $(\mathrm{Ni}(2+)$-requiring) | Glyceraldehyde-3-phosphate dehydrogenase (NADP(+)) $\operatorname{NAD}(\mathrm{P})(+)$ transhydrogenase (Re/Si-specific) Thymidine phosphorylase $P$ 2-oxopent-4-enoate hydratase | Alkanesulfonate monooxygenase $P$ Thiosulfate dehydrogenase P Sulfopropanediol 3-dehydrogenase $P$ Glutamate dehydrogenase $(\operatorname{NAD}(P)(+))$ Putrescine carbamoyltransferase $\mathbf{P}$ Dodecanoyl-[acyl-carrier-protein] hydrolase Glutathione synthase Nigerose phosphorylase P 2-methylisocitrate dehydratase $P$ Arginyltransferase $\boldsymbol{P}$

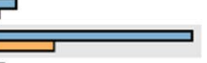

展

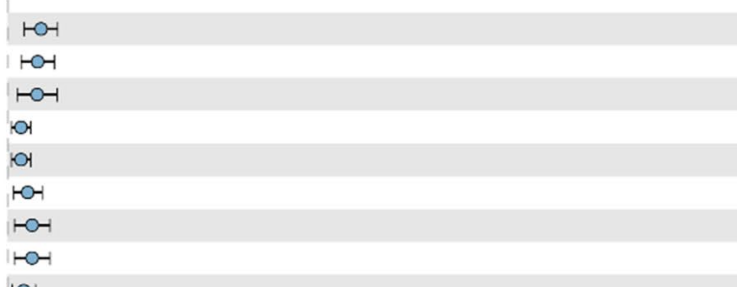

D hor

더
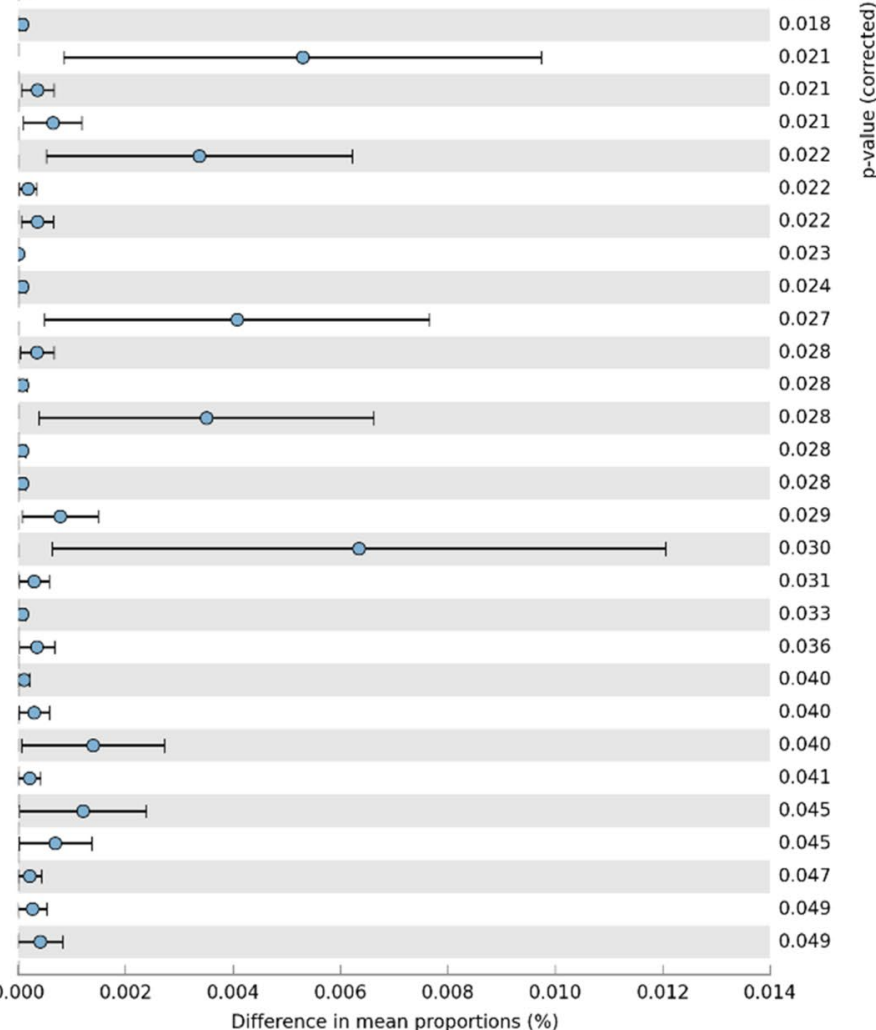

Figure 5. Significantly altered predicted functional KEGG enzymes at level 3 between high and low screen time groups. Single features displayed with $q$ value, effect size, and 95\% CI (Welch's $t$-test, $q<0.05$ ). Corrected $p$ values $(q)$ were calculated using Storey's FDR approach.

such as diet and lifestyle were predicted using 118 taxon sets. A network view of the least absolute shrinkage and selective operator (LASSO) results is provided in Supplementary Fig. S4. Compared to low screen time classification, subjects in the high screen time group had significantly greater abundance of taxon sets related to Crohn's disease $(q=0.001)$, type I diabetes $(q=0.003)$, having an overweight/obese mother $(q=0.003)$, and myocardial infarction $(q=0.008)$; high screen time users also had significantly greater abundance of taxa related to consumption of red wine $(q=0.007)$ and coffee $(q=0.025)$. Conversely, the low screen time group had significantly greater abundance of taxon sets related to liver cirrhosis $(q=0.001)$, autism $(q=0.006)$, and high-fat diet $(q=0.047)$, as compared to the high screen time group. 
a
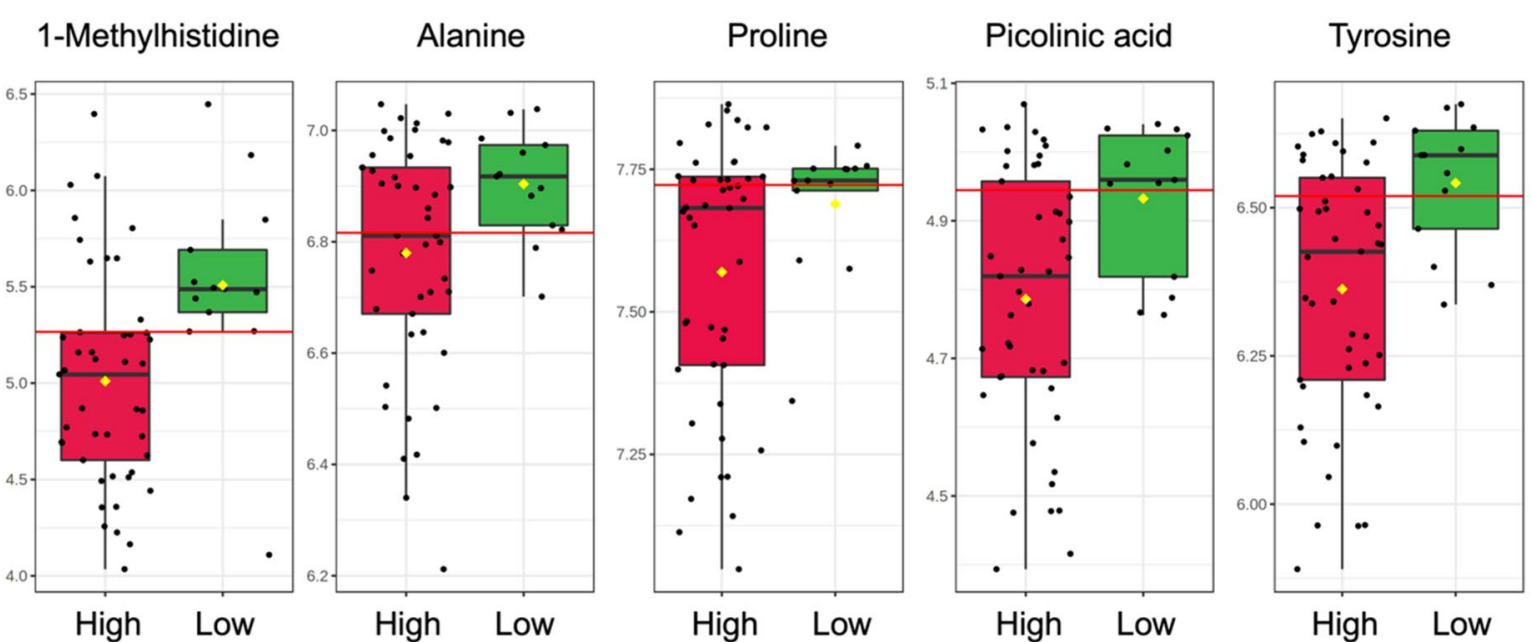

b

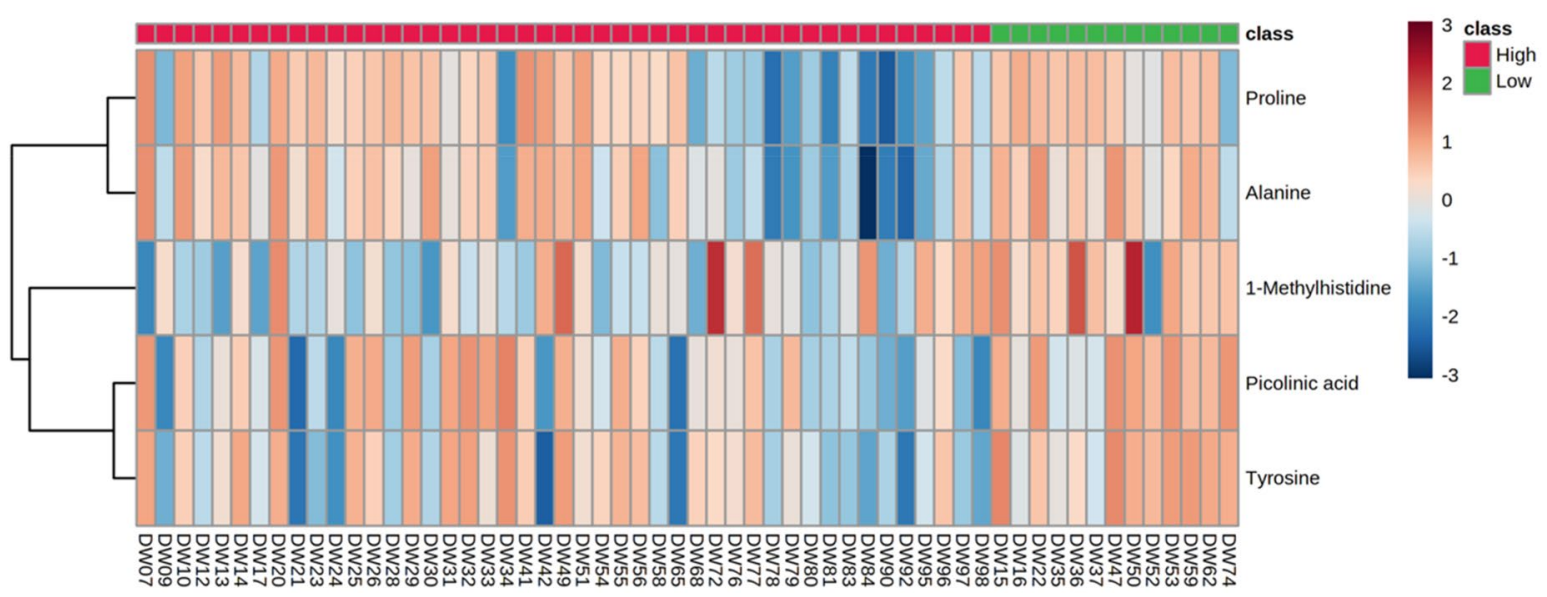

Figure 6. Significant metabolites between high and low screen time groups as determined by GLM with FDR correction. (a) Box plots of significant metabolites between groups showing normalized relative abundance. Age, sex, BMI, and MVPA were controlled for as covariates: 1-Methylhistidine $(q=0.002)$, Alanine $(q=0.020)$, Proline $(q=0.024)$, Picolinic acid $(q=0.034)$, Tyrosine $(q=0.048)$. Yellow diamonds signify group means, while horizontal red lines signify optimal between-group cut-offs. (b) Heatmap of significant metabolites by screen time classification.

Analysis of metabolomics data reveals significant differences in five metabolites between high and low screen time users. In total, 140 metabolites were reliably detected from human fecal samples using LC-MS/MS and GC-MS (quality control (QC) CV $<20 \%$, relative abundance $>1,000$ in $80 \%$ of samples). Relative levels of these 140 metabolites had a median coefficient of variation (CV) of $11.5 \%$, and $~ 76 \%$ of captured metabolites had QC CV $<15 \%$ (Supplementary Fig. S5). These metabolites spanned 20 different chemical classes and were representative of more than 35 metabolic pathways of potential biological relevance. All metabolomics data were log-transformed and Pareto scaled to approximate normality prior to analysis.

A general linear model (GLM) was used to assess differences in metabolite abundance between high and low screen time groups. Age, sex, BMI, and MVPA were controlled for as covariates, and significance was calculated with FDR correction. Results of the GLM showed five metabolites to be significantly decreased in the high screen time group: 1-methylhistidine $(q=0.002)$, alanine $(q=0.020)$, proline $(q=0.024)$, picolinic acid $(q=0.034)$, and tyrosine $(q=0.048)$ (Fig. 6a). A heatmap of significant metabolites by screen time classification is given in Fig. $6 \mathrm{~b}$. Full results of the GLM for all 140 reliably detected metabolites (including mean difference, standard error, FDR $q$, and 95\% confidence intervals), are displayed in Supplementary Table S3.

Predicted functional profile of the fecal metabolome differs by screen time. Enzyme enrichment analysis with LASSO regression was performed using 912 metabolic sets predicted to change in the case of dysfunctional enzymes (Supplementary Fig. S6). Seventeen enzymes were predicted to be significantly increased in the high screen time group (enrichment ratio $=8.0, p=0.003$ ). Two more enzymes, enolase and pyruvate kinase, were also predicted to be significantly increased in the high screen time group (enrichment ratio $=6.0$, $p=0.020$ ), while another three enzymes were significantly increased in high screen time by an enrichment ratio of 4.0: extracellular chitinase $(p=0.040)$, N-acetyl-D-glucosamine exchange $(p=0.040)$, and peroxisomal FAD 


\section{Enrichment Overview (top 25)}

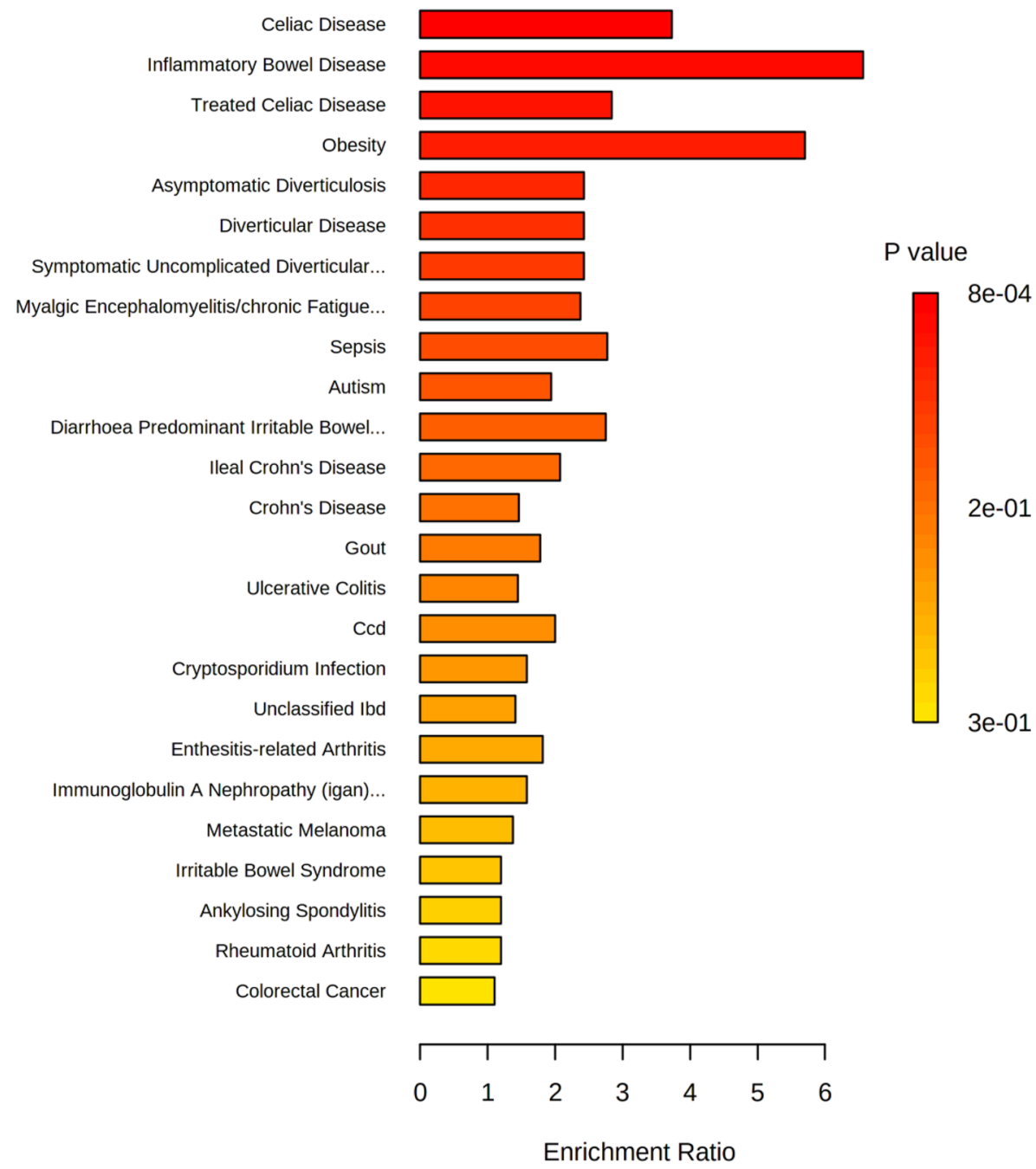

Figure 7. Enrichment analysis of disease signatures performed using 44 metabolite sets reported in human feces. Significant metabolite sets $(p)$ : celiac disease $(<0.001)$, inflammatory bowel disease $(0.001)$, treated celiac disease (0.008), obesity (0.016), asymptomatic diverticulitis (0.031), diverticular disease (0.031), symptomatic uncomplicated diverticular disease (0.031), and chronic fatigue syndrome (0.043).

transporter $(p=0.045)$. Supplementary Table S4 lists the complete set of enriched enzymes, along with their enrichment ratios and $p$ values.

Metabolomics data was also used to perform enrichment analysis of disease signatures using 44 metabolite sets reported in human feces (Fig. 7). Metabolites associated with eight disease signatures were significantly increased in the high screen time group: celiac disease $(p<0.001)$, inflammatory bowel disease $(p=0.001)$, treated celiac disease $(p=0.008)$, obesity $(p=0.016)$, asymptomatic diverticulitis $(p=0.031)$, diverticular disease $(p=0.031)$, symptomatic uncomplicated diverticular disease $(p=0.031)$, and chronic fatigue syndrome $(p=0.043)$.

Pathway topology analysis was conducted to predict pathway enrichment between study groups. Metabolomic data were mapped to the KEGG human pathway library, and significance and impact were calculated using a global test of relative-betweenness centrality. A scatter plot view of the results can be seen in Fig. 8 . Three pathways were observed to have both high impact $(\geq 0.50)$ and significance $(p<0.05)$ : phenylalanine (pathway impact $=1.0, p=0.008$ ), alanine, aspartate, and glutamate metabolism (pathway impact $=0.715, p=0.008$ ), and $\mathrm{D}$-glutamine/D-glutamate metabolism (pathway impact $=0.50, p=0.024$ ). All reliably detected metabolites in these pathways, with the exception of GABA, were decreased in the high screen time group.

Integrative analysis of multi-omics data revealed disturbances in amino acid metabolism. We used $\mathrm{mmvec}^{36}$ to integrate $16 \mathrm{~S}$ sequencing and metabolomic data to assess cooccurrence patterns between GM features (genera) and fecal metabolites. We found that Ruminococcaceae, previously identified by Songbird anal- 


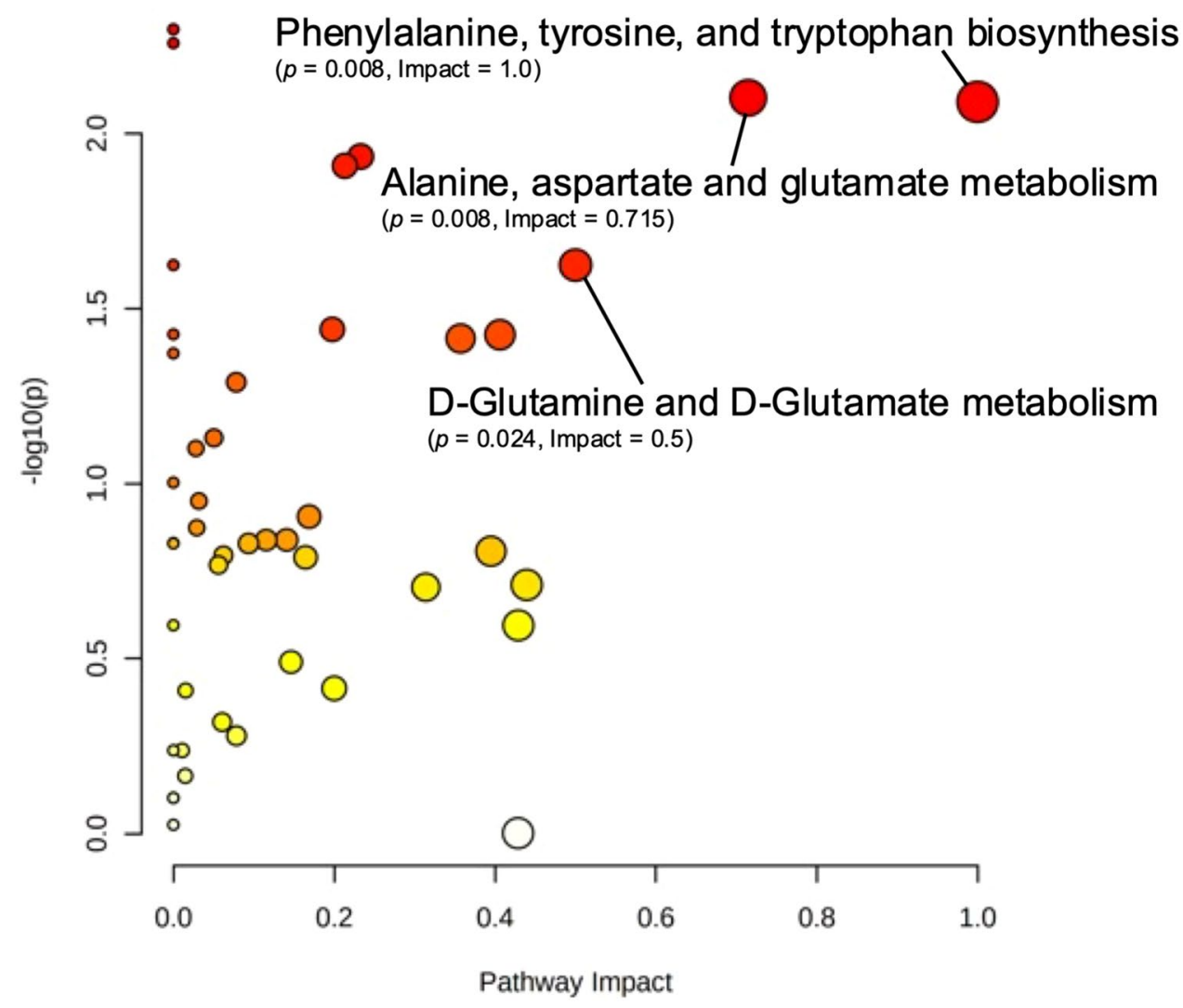

Figure 8. Scatter plot of pathway topology analysis. Metabolomic data were mapped to the KEGG human pathway library, and significance and impact were calculated using a global test of relative-betweenness centrality. Pathways with high impact $(\geq 0.50)$ and significance $(p<0.05)$ have been labeled.

ysis as the taxa most strongly associated with low screen time, had a high probability of co-occurring with five amino acids: isoleucine, L-alloisoleucine/leucine/norleucine, valine, proline, and phenylalanine. Upon visual examination of the biplot, we noted taxa with the highest cooccurrences were Collinsella, Lactobacillales, Ruminococcus, cc115 (family Erysipelotrichaceae), and Turicibacter (Supplementary Fig. S7). These taxa were also highly co-occurrent with the same five amino acids (Supplementary Table S5).

Predicted functional results were synthesized to form an integrated hypothesis describing the potential pathophysiology underlying high screen time. Our findings are conceptualized with respect to central carbon metabolism in Fig. 9. Three canonical KEGG pathways were indicated by pathway topology analysis as significantly impacted $(0.008 \leq p \leq 0.024)$ : (1) phenylalanine, tyrosine, and tryptophan biosynthesis, (2) alanine, aspartate, and glutamate metabolism, and (3) D-glutamine and D-glutamate metabolism. Between these pathways, 10 metabolites were detected in the current study; of these, six were significantly different between groups as determined by $t$-test with FDR correction $(0.006 \leq q \leq 0.042)$. Nine of these pathwayembedded metabolites were reduced in the high screen time group (alanine, asparagine, aspartate, fumarate, glutamate, glutamine, oxaloacetate, phenylalanine, tyrosine), while one metabolite was elevated with high screen time (GABA). Importantly, PICRUSt analysis predicted the mean proportions of two enzymes in these pathways to be significantly greater with high screen time. Namely, those were (S)-3-amino-2-methylpropionate transaminase $(q=6.07 \mathrm{e}-4)$ and glutamate dehydrogenase $(q=0.040)$. Additionally, when results of our mmvec analysis were incorporated into our hypothesis, a high co-occurrence of five bacterial species with one pathwayembedded metabolite (phenylalanine) was revealed. This indicates a high probability of microbe-metabolite interaction between phenylalanine and the subset of microbial consortia (C. collinsella, E. cc115, L. ruminococcus, Lactobacillales, and T. turicibacter). Higher abundance of all five bacteria was observed in the high screen time group. Box plots and significance information are included in Fig. 9 where appropriate.

\section{Discussion}

Screens are highly integrated in every facet of modern life, especially for young people ${ }^{1,2}$. Yet, despite growing concern over the detrimental effects of high screen time, previous studies have largely focused on the cognitive and behavioral effects of screen use and have exclusively relied on self-report data ${ }^{8-11}$, with only a few studies utilizing imaging techniques ${ }^{14-17}$. In this study, we explored the fecal microbiome and metabolome of a diverse 


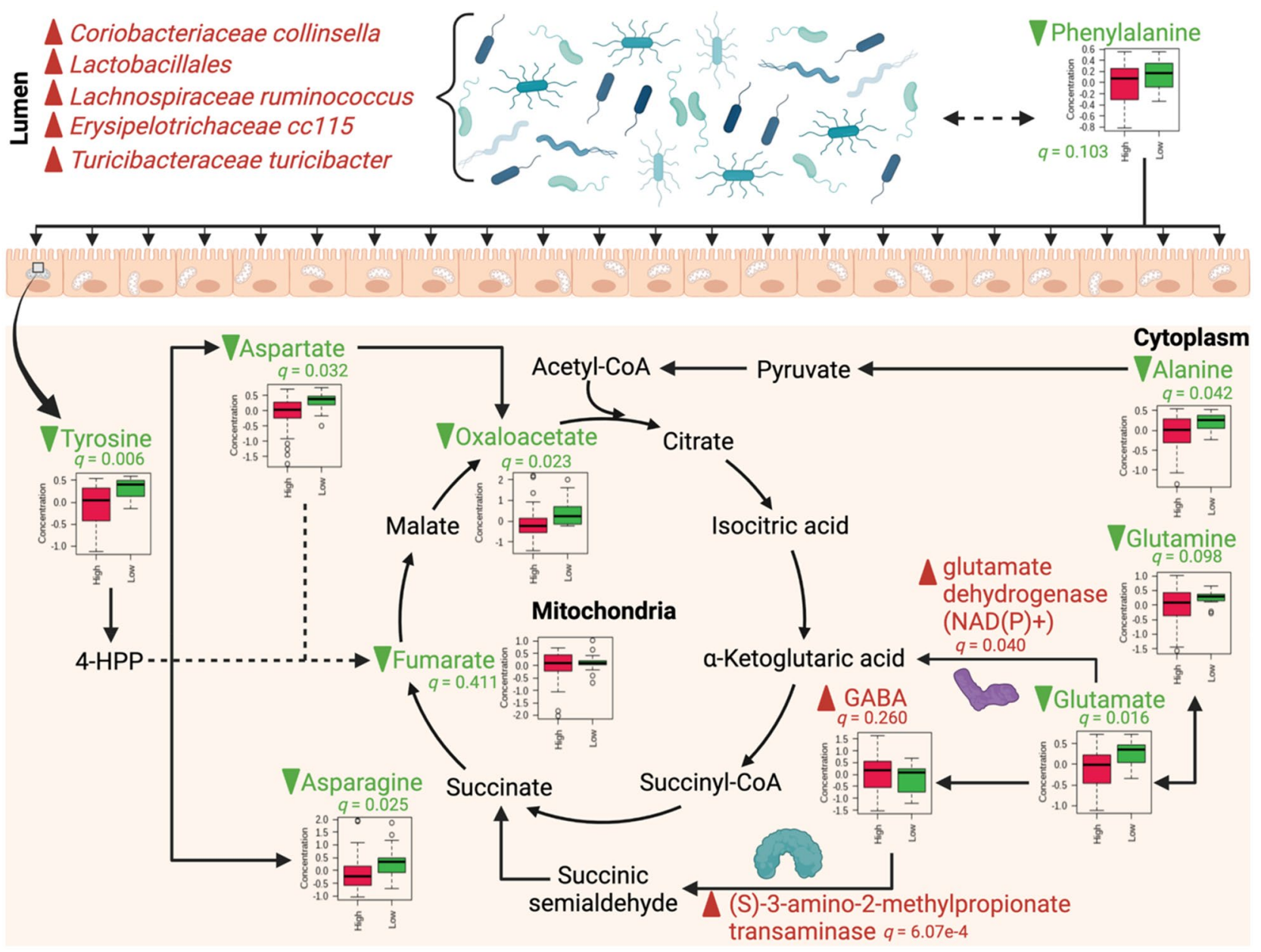

Figure 9. Integrated hypothesis of the potential pathophysiology underlying high screen time. The conceptual schema was synthesized using results of pathway topology analysis, PICRUSt analysis, and mmvec analysis. Green lettering with downward green arrows denotes decreases in the high screen time group, while red lettering and upward red arrows denotes increases in the high screen time group; box plots and significance information is provided where appropriate. Significance of plotted metabolites derived from independent $t$-test with FDR correction. Created with BioRender.com.

group of college students, classified by high ( $\geq 75 \mathrm{~min} /$ day) or low $(0-75 \mathrm{~min} /$ day $)$ screen time. The taxonomic profile of the microbiome, but not overall diversity, modestly differed between the two classifications of screen time. Indeed, several health-associated microbes such as Bacteroides, Akkermansia, Alistipes, Ruminococcus, Sutterella, Oscillospira, and Methanobrevibacter were found to be more associated with low screen time, even after accounting for physical activity, sex, BMI, and age. This finding suggests that compositional differences may occur based on daily screen time. Meanwhile, the metabolome proved to be a sensitive marker of screen time; a panel of five metabolites were significant lower in the high screen time group. Predicted functional analysis of the microbiome revealed significant enrichment of numerous enzymes and taxa associated with various disease, diet, and lifestyle factors. Predicted metabolomic states indicated enrichment of several other enzymes, pathways, and disease profiles. Cumulatively, our results revealed dysfunction of amino acid metabolism which related to several key taxa.

By comparing the log ratio abundances of screen-time associated taxa, we noted Ruminococcaceae was one of the features most associated with low screen time. This family of bacteria has been positively correlated with lower risk of weight gain and improved energy metabolism in mice ${ }^{37}$ and a reduction of metabolic syndrome in humans ${ }^{38}$. Against the top associated taxa with high screen time, we noted significance between log ratio abundances in some important instances. Specifically, a greater ratio of Peptostreptococcaceae/Runimococcaceae in those with a high screen time classification was observed. Peptostreptococcaceae has been found to be abundant in patients with bile duct and colorectal cancers ${ }^{39,40}$. In addition, the low screen time classification had a significantly greater Akkermansia/Bacteroides ratio. Akkermansia has been correlated to several health states ${ }^{41}$, such as being positively associated with an increase in insulin sensitivity ${ }^{42}$. More recently, the species Akkermansia muciniphila has been explored for use as an oral probiotic therapy for obesity and metabolic syndrome ${ }^{43}$. A genus that was found to be more associated with high screen time was Prevotella. This finding was intriguing, given that Prevotella has been considered beneficial due to its abundance in the GM of healthy individuals and associations with plant-rich diets and weight $\operatorname{loss}^{44,45}$. Moreover, Prevotella has been reported to be more common in 
non-Westernized populations ${ }^{46,47}$. However, Prevotella is a large genus with high species diversity and high genetic diversity between strains ${ }^{48}$. For example, several Prevotella species have been suggested as intestinal pathobionts ${ }^{49}$. Prevotella has also been found to be increased in individuals with metabolic syndrome ${ }^{50}$. Another ratio analyzed, Prevotella/Bacteroides, which have been promoted as potential biomarkers for diet and lifestyle ${ }^{51}$, did not show significance between classifications, suggesting that more evidence is needed to establish a predictive relationship.

We performed a prediction of function analysis using microbiome data, identifying significant differences in pathway enrichment between low and high screen time classification. The top differential predicted functions were significantly more enriched in the high screen time classification. These included enzymes involved in energy metabolism, such as CoA-transferases, carboxylases, and dehydrogenases. The most significant predicted function, glutaconate CoA-transferase, is responsible for producing acetate and glutaconyl-1-CoA ${ }^{52}$. Acetate may originate from microbial fermentation of residual peptides and fat ${ }^{53,54}$. In a Western diet with low fiber intake, protein fermentation occurs mainly in the distal colon when saccharolytic substrates have been depleted ${ }^{55}$. Participants in both classifications had fiber consumptions well below the AMDR. Increased protein fermentation can produce amino acids which can further be metabolized via cross-feeding mechanisms and alter gut integrity and insulin sensitivity ${ }^{56}$. Upon performing microbe-metabolite cooccurrence analysis we noted multiple amino acids with the great probability to occur with the taxa Collinsella, Lactobacillales, Ruminococcus, cc115, and Turicibacter. Although these did not differ significantly by screen time classification, they were all elevated in the high screen time group.

The metabolome also proved to differ by screen time. An age, sex, and BMI-controlled GLM showed significant disturbances $(q<0.05)$ in five metabolites: 1-methylhistidine, alanine, proline, picolinic acid, and tyrosine. Although no previous studies have shown any associations between screen time and levels of 1-methylhistidine, proline, picolinic acid or tyrosine, alanine has been previously linked to high screen time and increased metabolic risk factors ${ }^{57,58}$. Among adolescents, reduced alanine via increased alanine transaminase (ALT) activity was associated with high screen time, higher blood glucose, and reduced insulin sensitivity ${ }^{57}$. A similar association was observed between ALT and sedentary behavior in a cohort of obese 11-13-year-olds ${ }^{58}$. In the current study, levels of alanine and glutamate (both substrates and products of the reversible ALT reaction) were significantly lower in the high screen time group $(0.016 \leq q \leq 0.042)$. Future studies investigating objective markers of screen time for targeted therapy or surveillance monitoring should target the metabolome given its superior sensitivity compared to the more idiosyncratic changes of the microbiome.

Metabolome data was also mapped to canonical KEGG pathways to perform enzyme and pathway enrichment analyses. Additionally, database searches of fecal metabolites were conducted in order to construct predicted disease profiles with regard to screen time status. Three pathways were significantly enriched in response to screen time: (1) phenylalanine, tyrosine and tryptophan biosynthesis $(p=0.008),(2)$ alanine, aspartate and glutamate metabolism $(p=0.008)$, and (3) glutamate and glutamine metabolism $(p=0.024)$. This is the first reported association between screen time and differential enrichment of these pathways. Enzyme enrichment analysis returned 22 enzymes predicted to be significantly dysfunctional between groups, two of which were corroborated by PICRUSt analysis of microbiome data: glutamate dehydrogenase $(q=0.040)$ and (S)-3-amino-2-methylpropionate transaminase $(q=6.07 \mathrm{e}-4)$. The current study is the first to report predicted functional changes in these enzymes in response to high screen time. Analysis of predicted disease profiles showed significant enrichment of metabolites related to various gastrointestinal disorders such as celiac disease, inflammatory bowel disease, treated celiac disease, and various forms of diverticular disease $(0.001 \leq p \leq 0.031)$. Metabolite profiling of disease states also showed significant enrichment of metabolite sets related to obesity $(p=0.016)$ and chronic fatigue syndrome $(p=0.043)$. Many previous studies have demonstrated a strong, positive association between screen time and obesity risk and incidence ${ }^{4,5,10,11,17,57}$, while others have shown similarly strong associations between screen time, disordered sleep and fatigue ${ }^{4,5,8-10,17,57}$. Although no previous studies have indicated potential effects of screen time on GI diseases, many metabolites flagged as significant in the current study have been previously implicated in the onset and progression of GI disorders. For instance, levels of alanine and severity of celiac hepatitis are inversely correlated ${ }^{59}$, and proline catabolism has been shown to cause hypoxia and bowel inflammation ${ }^{60}$. Our results warrant further investigation into metabolite-driven changes in pathways relevant to GI disorders, with special regard to inflammatory conditions.

Cumulatively, we performed six discrete analyses of predicted functional changes in pathways and enzymes, as well as disease, diet, and lifestyle profiles. Notably, we also performed integrative analysis of microbiomic and metabolomic data using mmvec to identify cooccurrence patterns. Results of our pathway and enzyme enrichment analyses, PICRUSt analysis, and mmvec analysis are highly commensurate with one another and have been synthesized as an integrative hypothesis of predicted functional changes in Fig. 9. Interestingly, many changes predicted by our hypothesis have been previously implicated in disease, diet and lifestyle conditions noted by our TSEA and disease analysis of fecal metabolites. For instance, increased levels of Lactobacillales have been found in the stool of patients with chronic fatigue syndrome (CFS) ${ }^{61}$. In the current study, disease enrichment analysis predicted CFS to be significantly increased in the high screen time group (Fig. 7) and levels of Lactobacillales were also elevated in the high screen time group. Similarly, inverse associations between circulating levels of glutamate and tyrosine with clinical severity of CFS has previously been shown ${ }^{62}$; in our study, levels of both metabolites were significantly reduced in the high screen time group. Additionally, over-representation of Lachnospiraceae has been cited as a hallmark of inflammatory bowel conditions ${ }^{63,64}$. In our study, TSEA indicated significant enrichment of taxa related to Crohn's disease in the high screen time group (Supplementary Fig. S4) and disease enrichment of fecal metabolites revealed significant enrichment of metabolite sets linked to celiac disease, inflammatory bowel disease, and various forms of diverticular disease (Fig. 7).

While this explorative study provides the foundation for more directed research, it has some limitations, particularly that we had a relatively small number of participants $(n=60)$, who were categorized according to self-reported screen-time. Furthermore, 46 subjects were classified into high screen time, while 14 subjects were 
classified into low screen time. Future studies should make efforts to enroll more low screen time participants. Although the present study uses cross-sectional data with concurrent microbiomic and metabolomics data to explore actual and predicted effects of high screen time, future studies should validate these findings using longitudinal repeated-measures designs. Also, this study is limited to young, healthy college students living in the United States. Expanding this sample to a wider range of populations would allow us to capture a greater diversity of screen time, physical activity, and dietary data, and to better assess potential associations in microbial communities and metabolic signatures. Additionally, we did not produce strain-level resolution based on our sequencing methodology, though assessment at the species level in the future is warranted, as is metagenomic function.

Nevertheless, this is the first study to investigate the effects of screen time at the molecular level. We report the association of screen time with fecal microbiome and metabolome profiles in a cohort of healthy young college students. Significant features of these profiles may prove to be powerful therapeutic targets for the deleterious effects of increased screen time. Importantly, high screen time was found to be associated with reduced abundance of commensal bacteria Ruminococcaceae and Akkermansia. Moreover, the health-promoting taxa Bifidobacterium and Faecalibacterium were most predictive of low screen time. High screen time was predicted to have an increase in energy metabolism pathways, which may be suggestive of increased energy harvesting in the gut. Likewise, numerous metabolites associated with health in humans were significantly decreased in the high screen time group, suggesting an important role of the metabolome in the propagation of screen timeassociated pathogenesis. Importantly, we present an integrated hypothesis of widespread amino acid dysfunction and specific microbial-metabolite interactions using KEGG pathways, database searches, and advanced machine learning methods which deserves increased research given its ubiquitous indication in the current study.

\section{Methods}

Participants and study procedure. This study included data from 60 college students who were recruited from select on-campus dormitories at Arizona State University (ASU). Specifically, samples were obtained from a larger cross-sectional study that utilized mobile ecological momentary assessment methodology to assess the influence that social networks have on physical activity, dietary intake, and body weight in two residence halls at ASU in Tempe, Arizona ${ }^{65}$. Briefly, healthy college students living in on-campus housing, who were English speaking, and at least 18 years of age were eligible to participate. Exclusion criteria for this study included a history of malabsorptive disorders, high blood pressure, eating disorders, HIV infection, diabetes, and/or the use of antibiotics, antifungals, or probiotics in the 2 to 3 months prior to the study. This study was conducted during the Fall 2014 and Spring 2015 semesters. The ASU Institutional Review Board approved this study (STUDY00002019), and all participants provided written informed consent. All research protocols were conducted in accordance with the principles expressed in the Declaration of Helsinki.

Enrolled participants were provided with questionnaires capturing demographic, physical activity, and dietary intake data ${ }^{27,65}$. Height and weight were measured by trained research staff and BMI was calculated and participants were categorized based on the CDC guidelines ${ }^{66}$. Physical activity was assessed via the Godin-Shephard Leisure-Time Physical Activity Questionnaire ${ }^{67}$. Daily physical activity was calculated and MVPA was computed by summing the total time spent on strenuous and moderate activity. This validated survey for measuring physical activity habits in college-aged males and females also captured sedentary activity including screen time (excluding time in class and being physically active). Participants were asked to report average daily screen time categorically (i.e., 0-15 min, 15-75 min, 75-195 min, 195-315 min, 315-360 min, or 360 + min). Given previous screen time recommendations in children and adolescents by the $\mathrm{WHO}^{12}$ and the $\mathrm{AAP}^{13}$, as well deleterious effects associated with $120 \mathrm{~min}$ of screen time in adults ${ }^{2}$, we split participants at $75 \mathrm{~min}$ of self-reported screen time, as it conforms best to current knowledge and guidance. The ASA24 $24 \mathrm{~h}$ dietary recall was used to assess students' habitual dietary intake. Participants were also asked to complete three days of dietary recall (two weekdays and one weekend day). Days of intake were dropped if caloric intake was below 500 or in excess of $5000 \mathrm{kcal}$. If a participant did not have at least one day of adequate dietary intake they were excluded from the study. Using data from the ASA24-2014 Daily Total Nutrients Analysis File (TN), we examined total grams of protein, fat, carbohydrates, and fiber.

Sample collection. The sample collection procedure used in this study has been previously reported ${ }^{27}$. Each study participant was provided with a fecal sample collection kit (Commode Specimen Collection Kit, Fisher Scientific, Anthem, AZ) in order to provide a single fecal sample for analysis. Collection kits were distributed to participants in small insulated cooler bags containing ice packs to keep samples cold while in transit post-collection. Before participants left with the kit, a brief demonstration on how to collect the sample was provided along with a sheet of instructions inside the cooler bag. Participants were asked to freeze their ice packs immediately so that they were frozen at the time of sample collection. Ice packs were rated to stay frozen for $36-48 \mathrm{~h}$ in an insulated container. All stool samples were retrieved from participants and delivered to the clinical research facility within $24 \mathrm{~h}$ of collection. Stool samples were stored at $-80^{\circ} \mathrm{C}$ to preserve the microbial community.

Microbiome analysis. DNA isolation, preparation, and sequencing. Assessment of the intestinal microbiome from fecal collections was carried out at the Biodesign Institute at ASU in Tempe, Arizona. Extraction of microbial DNA from fecal samples was accomplished using the PowerSoil DNA isolation kit as described by the manufacturer (MoBio Laboratories Ltd., Carlsbad, CA) using a beadbeater (BioSpec, Bartlesville, OK). Amplification and sequencing of variants was performed as previously described ${ }^{27}$. 
Sequence data and statistical analysis. Raw 16S rRNA sequencing data for all samples have been deposited in the open-source repository "NCBI/Sequence Read Archive (SRA)" under project PRJNA473006 with accession numbers: SAMN09258197 - SAMN09258278 (https://www.ncbi.nlm.nih.gov/sra). In the present study, paired-end, demultiplexed data were imported and analyzed using QIIME 2 software version $2021.2^{68}$. Briefly, sequencing generated 5,259,656 reads with a median of 80,443 reads per sample. After viewing sequence quality plots based on 10,000 randomly selected reads, the first 13 bases of the forward and reverse reads were trimmed. Next, QC was performed via the DADA2 denoise-paired method to remove low quality regions and construct a feature table using amplicon sequence variants (ASVs). The feature-classifier plugin was used to classify ASVs taxonomically. A naive Bayes machine-learning classifier was pre-trained to differentiate taxa present in the 99\% Greengenes 13_8 reference set trimmed to 250 bp of the V4 hypervariable region (corresponding to the $515 \mathrm{~F}-806 \mathrm{R}$ primers) was used. This classifier works by identifying $k$-mers that are diagnostic for particular taxonomic groups and using that information to predict the taxonomic affiliation of each $\mathrm{ASV}^{69}$. Before constructing the taxonomic bar chart, we first filtered out any samples with fewer features than our rarefaction threshold $(10,000)$ and then filtered out low abundance/low prevalence ASVs using the feature-table plugin with the filter-samples method. A phylogenic tree was then constructed using the fragment-insertion plugin where the sequences were inserted into the Greengenes 13_8 99\% identity reference tree backbone. To account for uneven sequencing depth between samples, normalization was performed via alpha rarefaction. Based on the ASV feature table, a p-min-depth of 10 and a p-max-depth of 70,000 was used.

Diversity analysis was conducted with the diversity plugin. Alpha diversity (intra-community diversity) was measured using richness (Shannon, Faith's PD and observed ASVs) and evenness (Pielou) indexes and compared with Kruskal-Wallis tests. Beta diversity (inter-community diversity) was measured using Jaccard, Bray-Curtis, Unweighted UniFrac distance (qualitative measure), and Weighted UniFrac distance (quantitative measure). Adonis analyses with the covariates age, sex, MVPA, and BMI, were used to test for significant differences between high and low screen time for beta diversity metrics.

Relative abundance of taxa for high and low screen time classification were calculated at the genus level. The distinctive taxa between screen time classification were identified first through DEICODE, a compositionally aware form of Aitchison distance that is robust to high levels of sparsity ${ }^{30}$. This was conducted with the DEICODE plugin for QIIME 2 and the output was visualized as a robust Aitchison PCA, based on the feature table. To test significance using a multivariate model, the Adonis test in QIIME 2 was used to assess significant differences between screen time classification with the covariates sex, age, BMI, and MVPA time. Next, Songbird v1.0.1 $1^{31}$ in QIIME 2 version 2020.6 was used to identify feature ranks (parameters: - p-epochs 10,000 - p-differential-prior 0.5 - p-summary-interval 1 - num-random-test-examples $10 \%$ of samples) based on screen time and accounting for the covariates sex, BMI, age, and MVPA time. Qurro v0.4. $0^{32}$ was then used to compute log ratios of ranked features. Evaluation of the Songbird models against a baseline model obtained a pseudo- $\mathrm{Q}^{2}$ value of -0.102 , suggestive of possible overfitting. The top 20 highest and lowest ranked differential features were selected based on screen time classification. T-tests (Mann-Whitney $U$ tests) and Cohen's $d$ were calculated to assess the significance $($ alpha $=0.05)$ and effect size of the log ratios.

In order to predict the function of fecal microbiota, data analysis was performed through the PICRUSt 2 pipeline $^{33}$. Then, PICRUSt output for the level 3 KEGG entries were analyzed and illustrated with statistical analysis of the taxonomic and functional profiles (STAMP) software version 2.1.3 $3^{70}$. Welch's $t$-test was used to test for significant features between screen time classification with $p$-value corrections performed using Storey's FDR approach.

Taxon set enrichment analysis (TSEA) of disease, diet and lifestyle, in addition to core statistical and visual analysis of microbiome data, was performed using MicrobiomeAnalyst v1.0 ${ }^{35}$.

Metabolomics analysis. Reagents. Acetonitrile (ACN), methanol $(\mathrm{MeOH})$, and ammonium acetate $\left(\mathrm{NH}_{4} \mathrm{Oac}\right)$, all LC-MS grade, were purchased from Fisher Scientific (Pittsburgh, PA). Ammonium hydroxide $\left(\mathrm{NH}_{4} \mathrm{OH}\right)$, O-methylhydroxylamine hydrochloride (MeOX), and N-Methyl-n-(tert-butyldimethylsilyl) trifluoroacetamide (MTBSTFA) were bought from Sigma-Aldrich (Saint Louis, MO). Deionized water was provided in-house by a water purification system from EMD Millipore (Billerica, MA). Phosphate buffered saline (PBS) was bought from GE Healthcare Life Sciences (Logan, UT). Standard compounds corresponding to measured aqueous metabolites/features were purchased from Sigma-Aldrich and Fisher Scientific. Lipid standards were purchased from Fisher Scientific, Sigma-Aldrich, and Avanti Polar Lipids (Alabaster, AL).

Targeted LC-MS/MS aqueous profiling. Prior to LC-MS/MS targeted measurement, frozen fecal samples were first thawed overnight under $4{ }^{\circ} \mathrm{C}$. Afterward, $20 \mathrm{mg}$ of each sample were placed in a $2 \mathrm{~mL}$ Eppendorf vial. The initial step for protein precipitation and metabolite extraction was performed by adding $500 \mu \mathrm{L} \mathrm{MeOH}$ and 50 $\mu \mathrm{L}$ internal standard solution (containing $1,810.5 \mu \mathrm{M}{ }^{13} \mathrm{C}_{3}$-lactate and $142 \mu \mathrm{M}^{13} \mathrm{C}_{5}$-glutamic acid). The mixture was then vortexed for $10 \mathrm{~s}$ and stored at $-20^{\circ} \mathrm{C}$ for $30 \mathrm{~min}$, followed by centrifugation at $14,000 \mathrm{RPM}(21,913 \times g)$ for $10 \mathrm{~min}$ at $4{ }^{\circ} \mathrm{C}$. The supernatants $(450 \mu \mathrm{L})$ were collected into new Eppendorf vials and dried using a CentriVap Concentrator (Fort Scott, KS). The dried samples were reconstituted in $150 \mu \mathrm{L}$ of $40 \% \mathrm{PBS} / 60 \% \mathrm{ACN}$ and centrifuged again at $14,000 \mathrm{RPM}(21,913 \times \mathrm{g})$ at $4{ }^{\circ} \mathrm{C}$ for $10 \mathrm{~min}$. Afterward, $100 \mu \mathrm{L}$ of supernatant was collected from each sample into an LC autosampler vial for subsequent analysis. A pooled sample, which was a mixture of all experimental samples, was used as the QC sample and injected once every 10 experimental samples.

The targeted LC-MS/MS method used here was modeled after that developed and used in a growing number of studies ${ }^{71-73}$. Briefly, all LC-MS/MS experiments were performed on an Agilent 1290 UPLC-6490 QQQ-MS system. Each supernatant sample was injected twice, $10 \mu \mathrm{L}$ for analysis using negative ionization mode and $4 \mu \mathrm{L}$ for analysis using positive ionization mode. Both chromatographic separations were performed in hydrophilic 
interaction chromatography mode on a Waters Xbridge BEH Amide column $(150 \times 2.1 \mathrm{~mm}, 2.5 \mu \mathrm{m}$ particle size; Waters Corporation, Milford, MA). The flow rate was $0.3 \mathrm{~mL} / \mathrm{min}$, auto-sampler temperature was kept at $4{ }^{\circ} \mathrm{C}$, and the column compartment was set to $40^{\circ} \mathrm{C}$. The mobile phase was composed of Solvents A (10 $\mathrm{mM} \mathrm{NH}_{4} \mathrm{Oac}$, $10 \mathrm{mM} \mathrm{NH}_{4} \mathrm{OH}$ in $\left.95 \% \mathrm{H}_{2} \mathrm{O} / 5 \% \mathrm{ACN}\right)$ and $\mathrm{B}\left(10 \mathrm{mM} \mathrm{NH}_{4} \mathrm{Oac}, 10 \mathrm{mM} \mathrm{NH} 4 \mathrm{OH}\right.$ in $\left.95 \% \mathrm{ACN} / 5 \% \mathrm{H}_{2} \mathrm{O}\right)$. After an initial $1 \mathrm{~min}$ isocratic elution of $90 \% \mathrm{~B}$, the percentage of Solvent B decreased to $40 \%$ at $\mathrm{t}=11 \mathrm{~min}$. The composition of Solvent B was maintained at $40 \%$ for $4 \mathrm{~min}(t=15 \mathrm{~min})$, after which the percentage of B gradually went back to $90 \%$, to prepare for the next injection. The mass spectrometer was equipped with an electrospray ionization (ESI) source. Targeted data acquisition was performed in multiple-reaction-monitoring (MRM) mode. For targeted data acquisition, we monitored 118 and 160 MRM transitions in negative and positive mode, respectively (278 transitions in total). The whole LC-MS system was controlled by Agilent MassHunter Workstation software. The extracted MRM peaks were integrated using Agilent MassHunter Quantitative Data Analysis software.

GC-MS analysis of short chain fatty acids (SCFAs). Frozen fecal samples were first thawed overnight under $4{ }^{\circ} \mathrm{C}$. Afterward, $20 \mathrm{mg}$ of each sample was homogenized with $5 \mu \mathrm{L}$ hexanoic acid-3,3,3 (internal standard), 15 $\mu \mathrm{L}$ sodium hydroxide $(\mathrm{NaOH}[0.5 \mathrm{M}])$, and $500 \mu \mathrm{L} \mathrm{MeOH}$. Following storage at $-20^{\circ} \mathrm{C}$ for $20 \mathrm{~min}$ and centrifugation at $14,000 \mathrm{RPM}(21,913 \times g)$ for $10 \mathrm{~min}, 450 \mu \mathrm{L}$ of supernatant were collected and sample $\mathrm{pH}$ was adjusted to 10 by adding $30 \mu \mathrm{L}$ of $\mathrm{NaOH}: \mathrm{H}_{2} \mathrm{O}(1: 4, \mathrm{v}: \mathrm{v})$. Samples were then dried, and the residues were first derivatized with $40 \mu \mathrm{L}$ of $20 \mathrm{mg} / \mathrm{mL}$ MeOX solution in pyridine under $60^{\circ} \mathrm{C}$ for $90 \mathrm{~min}$. Next, $60 \mu \mathrm{L}$ of MTBSTFA containing $\mathrm{d}_{27}$-mysristic acid were added, and the mixture was incubated at $60^{\circ} \mathrm{C}$ for $30 \mathrm{~min}$. The samples were then vortexed for $30 \mathrm{~s}$, followed by centrifugation at $14,000 \mathrm{RPM}(21,913 \times \mathrm{g})$ for $10 \mathrm{~min}$. Finally, $70 \mu \mathrm{L}$ of supernatant were collected from each sample into new glass vials for GC-MS analysis.

GC-MS conditions used here were adopted from a previously published protocol ${ }^{74}$. Briefly, GC-MS experiments were performed on an Agilent 7820A GC-5977B MSD system (Santa Clara, CA) by injecting $1 \mu \mathrm{L}$ of prepared samples. Helium was used as the carrier gas with a constant flow rate of $1.2 \mathrm{~mL} / \mathrm{min}$. The separation of metabolites was achieved using an Agilent HP-5 ms capillary column $(30 \mathrm{~m} \times 250 \mu \mathrm{m} \times 0.25 \mu \mathrm{m})$. The column temperature was maintained at $60^{\circ} \mathrm{C}$ for $1 \mathrm{~min}$, increased at a rate of $10^{\circ} \mathrm{C} / \mathrm{min}$ to $325^{\circ} \mathrm{C}$, and then held at this temperature for $10 \mathrm{~min}$. Mass spectral signals were recorded at an $\mathrm{m} / z$ range of 50-600. Data extraction was performed using Agilent MassHunter Profinder software. A batch recursive feature extraction algorithm for small molecules was used, and peaks were filtered so that only peaks with absolute height $\geq 1,000$ counts were included. An RT tolerance of 0.10 min was established, and extraction was limited to the largest 1,000 compound groups. Results were filtered if the overall identification score was less than 75 .

Metabolite data analysis. Following peak integration, metabolites were filtered for reliability and only those with QC CV $<20 \%$ and relative abundance of 1000 in $>80 \%$ of samples were retained for analysis. The data were $\log _{10}$-transformed and Pareto scaled prior to analysis. Linear modelling was performed using SPSS 28.0 (SPSS Inc., Chicago, IL). Multivariate statistical analyses were performed using open-source R software. Pathway and enzyme enrichment analysis of metabolomic data were performed and visualized using MetaboAnalyst v5.075.

Multi-omics data analysis. In order to identify microbial features associated with screen time classification and the metabolites they might be producing, we measured probabilities of cooccurrence between observed species (based on metagenomic data) and all metabolites (as informed by the metabolomic analysis). For this analysis, we used mmvec v1.0.2, a neural network solution inspired from natural language processing, to build a log-transformed conditional probability matrix from each cross-omics feature pair and apply singular value decomposition in order to represent cooccurrence in the form of biplots ${ }^{76}$.

\section{Data availability}

Raw 16S rRNA sequencing data for all samples have been deposited in the open-source repository "NCBI/ Sequence Read Archive (SRA)" under project PRJNA473006 with accession numbers: SAMN09258197SAMN09258278 (https://www.ncbi.nlm.nih.gov/sra). All mass spectrometry data and deidentified subject metadata analyzed in this study have been deposited to Mendeley Data and are publicly available (https://doi. $\operatorname{org} / 10.17632 /$ nd64f8zchj.1).

Received: 1 December 2021; Accepted: 11 February 2022

Published online: 02 March 2022

\section{References}

1. Lancet, T. Social media, screen time, and young people's mental health. Lancet 393, 611 (2019).

2. Orben, A. \& Przybylski, A. K. The association between adolescent well-being and digital technology use. Nat. Hum. Behav. 3, 173-182 (2019).

3. Dickson, K. et al. Screen-Based Activities and Children and Young People's Mental Health and Psychosocial Wellbeing: A Systematic Map of Reviews. http://eppi.ioe.ac.uk/ (2019).

4. Duch, H., Fisher, E. M., Ensari, I. \& Harrington, A. Screen time use in children under 3 years old: A systematic review of correlates. Int. J. Behav. Nutr. Phys. Act. 10, 102 (2013).

5. Magee, C. A., Lee, J. K. \& Vella, S. A. Bidirectional relationships between sleep duration and screen time in early childhood. JAMA Pediatr. 168, 465-470 (2014).

6. Anderson, K. Internet use among college students: An exploratory study. J. Am. Coll. Heal. 50, 21-26 (2001).

7. Fountaine, C. J., Liguori, G. A., Mozumdar, A. \& Schuna, J. M. Physical activity and screen time sedentary behaviors in college students. Int. J. Exerc. Sci. 4, 102-112 (2011).

8. Madigan, S., Browne, D., Racine, N., Mori, C. \& Tough, S. Association between screen time and children's performance on a developmental screening test. JAMA Pediatr. 173, 244-250 (2019). 
9. Boers, E., Afzali, M. H., Newton, N. \& Conrod, P. Association of screen time and depression in adolescence. JAMA Pediatr. 173, $853-859$ (2019).

10. Janssen, X. et al. Associations of screen time, sedentary time and physical activity with sleep in under $5 \mathrm{~s}$ : A systematic review and meta-analysis. Sleep Med. Rev. 49, 101226 (2020).

11. Tebar, W. R. et al. Increased screen time is associated with alcohol desire and sweetened foods consumption during the COVID-19 pandemic. Front. Nutr. 8, 630586 (2021).

12. World Health Organization. Guidelines on Physical Activity, Sedentary Behaviour and Sleep for Children under 5 Years of Age (WHO, 2019).

13. Council on Communications and Media. Media and young minds. Pediatrics 138, e20162591 (2016).

14. Hutton, J. S., Dudley, J., Horowitz-Kraus, T., DeWitt, T. \& Holland, S. K. Associations between screen-based media use and brain white matter integrity in preschool-aged children. JAMA Pediatr. 174, e193869 (2020).

15. Horowitz-Kraus, T. \& Hutton, J. S. Brain connectivity in children is increased by the time they spend reading books and decreased by the length of exposure to screen-based media. Acta Paediatr. 107, 685-693 (2018).

16. Rodriguez-Ayllon, M. et al. Associations of physical activity and screen time with white matter microstructure in children from the general population. Neuroimage 205, 116258 (2020).

17. Domingues-Montanari, S. Clinical and psychological effects of excessive screen time on children. J. Paediatr. Child Health 53, $333-338$ (2017).

18. Huttenhower, C. et al. Structure, function and diversity of the healthy human microbiome. Nature 486, 207-214 (2012).

19. Gupta, S. et al. Amplicon sequencing provides more accurate microbiome information in healthy children compared to culturing. Commun. Biol. 2, 1-7 (2019).

20. Sanschagrin, S. \& Yergeau, E. Next-generation sequencing of 16 S ribosomal RNA gene amplicons. J. Vis. Exp. 90, e51709 (2014).

21. Qin, J. et al. A human gut microbial gene catalogue established by metagenomic sequencing. Nature 464, 59-65 (2010).

22. Wikoff, W. R. et al. Metabolomics analysis reveals large effects of gut microflora on mammalian blood metabolites. Proc. Natl. Acad. Sci. U. S. A. 106, 3698-3703 (2009).

23. Visconti, A. et al. Interplay between the human gut microbiome and host metabolism. Nat. Commun. 10, 4505 (2019).

24. Wilmanski, T. et al. Blood metabolome predicts gut microbiome $\alpha$-diversity in humans. Nat. Biotechnol. 37, 1217-1228 (2019).

25. Krautkramer, K. A., Fan, J. \& Bäckhed, F. Gut microbial metabolites as multi-kingdom intermediates. Nat. Rev. Microbiol. 19, 77-94 (2021).

26. Lamichhane, S., Sen, P., Dickens, A. M., Orešič, M. \& Bertram, H. C. Gut metabolome meets microbiome: A methodological perspective to understand the relationship between host and microbe. Methods 149, 3-12 (2018).

27. Whisner, C., Maldonado, J., Dente, B., Krajmalnik-Brown, R. \& Bruening, M. Diet, physical activity and screen time but not body mass index are associated with the gut microbiome of a diverse cohort of college students living in university housing: A crosssectional study. BMC Microbiol. 18, 210 (2018).

28. Nelson, M. C., Story, M., Larson, N. I., Neumark-Sztainer, D. \& Lytle, L. A. Emerging adulthood and college-aged youth: An overlooked age for weight-related behavior change. Obesity 16, 2205-2211 (2008).

29. Yatsunenko, T. et al. Human gut microbiome viewed across age and geography. Nature 486, 222-227 (2012).

30. Martino, C. et al. A novel sparse compositional technique reveals microbial perturbations. mSystems 4, 5. https://doi.org/10.1128/ mSystems.00016-19 (2019).

31. Morton, J. T. et al. Establishing microbial composition measurement standards with reference frames. Nat. Commun. 10, 1-11 (2019).

32. Fedarko, M. W. et al. Qurro. https://doi.org/10.5281/ZENODO.3369454 (2019).

33. Douglas, G. M. et al. PICRUSt2 for prediction of metagenome functions. Nat. Biotechnol. 38, 685-688 (2020).

34. Kanehisa, M. \& Goto, S. KEGG: Kyoto encyclopedia of genes and genomes. Nucleic Acids Res. 28, 27-30 (2000).

35. Chong, J., Liu, P., Zhou, G. \& Xia, J. Using MicrobiomeAnalyst for comprehensive statistical, functional, and meta-analysis of microbiome data. Nat. Protoc. 15, 799-821 (2020).

36. Buas, M. F. et al. Identification of novel candidate plasma metabolite biomarkers for distinguishing serous ovarian carcinoma and benign serous ovarian tumors. Gynecol. Oncol. 140, 138-144 (2016).

37. Menni, C. et al. Gut microbiome diversity and high-fibre intake are related to lower long-term weight gain. Int. J. Obes. 41, 1099-1105 (2017).

38. Wu, X., Unno, T., Kang, S. \& Park, S. A Korean-style balanced diet has a potential connection with ruminococcaceae enterotype and reduction of metabolic syndrome incidence in Korean adults. Nutrients 13, 495 (2021).

39. Ahn, J. et al. Human gut microbiome and risk for colorectal cancer. J. Natl. Cancer Inst. 105, 1907-1911 (2013).

40. Jia, X. et al. Characterization of gut microbiota, bile acid metabolism, and cytokines in intrahepatic cholangiocarcinoma. Hepatology 71, 893-906 (2020).

41. Naito, Y., Uchiyama, K. \& Takagi, T. A next-generation beneficial microbe: Akkermansia muciniphila. J. Clin. Biochem. Nutr. 63, 35 (2018).

42. Shin, N. R. et al. An increase in the Akkermansia spp. population induced by metformin treatment improves glucose homeostasis in diet-induced obese mice. Gut 63, 727-735 (2014).

43. Depommier, C. et al. Supplementation with Akkermansia muciniphila in overweight and obese human volunteers: A proof-ofconcept exploratory study. Nat. Med. 25, 1096-1103 (2019).

44. Precup, G. \& Vodnar, D. C. Gut Prevotella as a possible biomarker of diet and its eubiotic versus dysbiotic roles: a comprehensive literature review. Br. J. Nutr. 122, 131-140 (2019).

45. Christensen, L. et al. Prevotella abundance predicts weight loss success in healthy, overweight adults consuming a whole-grain diet ad libitum: A post hoc analysis of a 6-wk randomized controlled trial. J. Nutr. 149, 2174-2181 (2019).

46. Wu, G. D. et al. Linking long-term dietary patterns with gut microbial enterotypes. Science 334, 108 (2011).

47. Filippo, C. D. et al. Impact of diet in shaping gut microbiota revealed by a comparative study in children from Europe and rural Africa. Proc. Natl. Acad. Sci. U. S. A. 107, 14691-14696 (2010).

48. Gupta, V. K., Chaudhari, N. M., Iskepalli, S. \& Dutta, C. Divergences in gene repertoire among the reference Prevotella genomes derived from distinct body sites of human. BMC Genom. 16, 153 (2015).

49. Larsen, J. M. The immune response to Prevotella bacteria in chronic inflammatory disease. Immunology 151, 363-374 (2017).

50. Guevara-Cruz, M. et al. Improvement of lipoprotein profile and metabolic endotoxemia by a lifestyle intervention that modifies the gut microbiota in subjects with metabolic syndrome. J. Am. Heart Assoc. 8, e012401 (2019).

51. Gorvitovskaia, A., Holmes, S. P. \& Huse, S. M. Interpreting Prevotella and Bacteroides as biomarkers of diet and lifestyle. Microbiome 4, 15 (2016).

52. Buckel, W., Dorn, U. \& Semmler, R. Glutaconate CoA-transferase from Acidaminococcus fermentans. Eur. J. Biochem. 118, 315-321 (1981).

53. Mortensen, P. B., Holtug, K., Bonnén, H. \& Clausen, M. R. The degradation of amino acids, proteins, and blood to short-chain fatty acids in colon is prevented by lactulose. Gastroenterology 98, 353-360 (1990).

54. Koh, A., De Vadder, F., Kovatcheva-Datchary, P. \& Bäckhed, F. From dietary fiber to host physiology: Short-chain fatty acids as key bacterial metabolites. Cell 165, 1332-1345 (2016). 
55. Blachier, F., Mariotti, F., Huneau, J. F. \& Tomé, D. Effects of amino acid-derived luminal metabolites on the colonic epithelium and physiopathological consequences. Amino Acids 33, 547-562 (2007).

56. Windey, K., De Preter, V. \& Verbeke, K. Relevance of protein fermentation to gut health. Mol. Nutr. Food Res. 56, 184-196 (2012).

57. Hardy, L. L., Denney-Wilson, E., Thrift, A. P., Okely, A. D. \& Baur, L. A. Screen time and metabolic risk factors among adolescents. Arch. Pediatr. Adolesc. Med. 164, 643-649 (2010).

58. Norman, G. J. et al. Sedentary behavior and cardiometabolic health associations in obese 11-13-year olds. Child. Obes. 13, 425-432 (2017).

59. Rubio-Tapia, A. \& Murray, J. The liver and celiac disease. Clin. Liver Dis. 23, 167-176 (2019).

60. Eltzschig, H. \& Carmeliet, P. Hypoxia and inflammation. N. Engl. J. Med. 364, 656-665 (2011).

61. Galland, L. The gut microbiome and the brain. J. Med. Food 17, 1272 (2014).

62. Naviaux, R. K. et al. Metabolic features of chronic fatigue syndrome. Proc. Natl. Acad. Sci. U. S. A. 113, E5480 (2016)

63. Halfvarson, J. et al. Dynamics of the human gut microbiome in inflammatory bowel disease. Nat. Microbiol. 2, 17004 (2017).

64. Clemente, J. C., Manasson, J. \& Scher, J. U. State of the Art Review: The role of the gut microbiome in systemic inflammatory disease. BMJ 360, j5145 (2018).

65. Bruening, M. et al. A mobile ecological momentary assessment tool (devilSPARC) for nutrition and physical activity behaviors in college students: A validation study. J. Med. Internet Res. 18, e209 (2016).

66. NHLBI Obesity Education Initiative Expert Panel on the Identification, Evaluation, and T. of O. in A. (US). Clinical Guidelines on the Identification, Evaluation, and Treatment of Overweight and Obesity in Adults. https://www.ncbi.nlm.nih.gov/books/NBK20 03/ (1998).

67. Godin, G. \& Shephard, R. J. A simple method to assess exercise behavior in the community. Can. J. Appl. Sport Sci. 10, 141-146 (1985).

68. Bolyen, E. et al. Reproducible, interactive, scalable and extensible microbiome data science using QIIME 2. Nat. Biotechnol. 37, 852-857 (2019).

69. Bokulich, N. A. et al. Optimizing taxonomic classification of marker-gene amplicon sequences with QIIME 2's q2-feature-classifier plugin. Microbiome 6, 1-17 (2018).

70. Parks, D. H., Tyson, G. W., Hugenholtz, P. \& Beiko, R. G. STAMP: Statistical analysis of taxonomic and functional profiles. Bioinformatics 30, 3123-3124 (2014).

71. Jasbi, P. et al. Daily red wine vinegar ingestion for eight weeks improves glucose homeostasis and affects the metabolome but does not reduce adiposity in adults. Food Funct. 10, 7343-7355 (2019).

72. Jasbi, P. et al. Coccidioidomycosis detection using targeted plasma and urine metabolic profiling. J. Proteome Res. 18, 2791-2802 (2019).

73. Jasbi, P. et al. Metabolic profiling of neocortical tissue discriminates Alzheimer's disease from mild cognitive impairment, high pathology controls, and normal controls. J. Proteome Res. 20, 4303-4317 (2021).

74. Gu, H., Jasbi, P., Patterson, J. \& Yan, J. Enhanced detection of short-chain fatty acids using gas chromatography mass spectrometry. Curr. Protoc. 1, e177 (2021).

75. Pang, Z. et al. MetaboAnalyst 5.0: Narrowing the gap between raw spectra and functional insights. Nucleic Acids Res. 49, W388W396 (2021).

76. Morton, J. T. et al. Learning representations of microbe-metabolite interactions. Nat. Methods 16, 1306-1314 (2019).

\section{Acknowledgements}

This study was supported by the NIH Common Fund from the Office of the Director and the Office of Behavioral and Social Sciences Research, grant number 1DP5OD017910 (PI: M. Bruening). The content is solely the responsibility of the authors and does not necessarily represent the official views of the National Institute of Health.

\section{Author contributions}

Conceptualization: P.J., M.B., H.G. and C.W.; Data curation: P.J., A.E.M., X.S., T.M., M.B. and C.W.; Formal analysis: P.J. and A.E.M.; Funding acquisition: M.B.; Investigation: X.S., T.M., M.B. and C.W.; Methodology: P.J., A.E.M., M.B., H.G. and C.W.; Project administration: P.J., A.E.M., M.B., H.G. and C.W.; Resources: M.B., H.G. and C.W.; Software: P.J., A.E.M. and Q.Z.; Supervision: Q.Z., M.B., H.G. and C.W.; Validation: P.J. and A.E.M.; Visualization: P.J. and A.E.M.; Writing—original draft: P.J. and A.E.M.; Writing—review \& editing: P.J., A.E.M., Q.Z., M.B., H.G. and C.W.

\section{Competing interests}

The authors declare no competing interests.

\section{Additional information}

Supplementary Information The online version contains supplementary material available at https://doi.org/ 10.1038/s41598-022-07381-3.

Correspondence and requests for materials should be addressed to H.G. or C.W.

Reprints and permissions information is available at www.nature.com/reprints.

Publisher's note Springer Nature remains neutral with regard to jurisdictional claims in published maps and institutional affiliations.

(c) (1) Open Access This article is licensed under a Creative Commons Attribution 4.0 International License, which permits use, sharing, adaptation, distribution and reproduction in any medium or format, as long as you give appropriate credit to the original author(s) and the source, provide a link to the Creative Commons licence, and indicate if changes were made. The images or other third party material in this article are included in the article's Creative Commons licence, unless indicated otherwise in a credit line to the material. If material is not included in the article's Creative Commons licence and your intended use is not permitted by statutory regulation or exceeds the permitted use, you will need to obtain permission directly from the copyright holder. To view a copy of this licence, visit http://creativecommons.org/licenses/by/4.0/.

(c) The Author(s) 2022 